\title{
Dualising consistent IIA/IIB truncations
}

\author{
Emanuel Malek ${ }^{a}$ and Henning Samtleben ${ }^{b}$ \\ ${ }^{a}$ Arnold Sommerfeld Center for Theoretical Physics, Department für Physik, \\ Ludwig-Maximilians-Universität München, Theresienstraße 37, München, 80333 Germany \\ ${ }^{b}$ Université de Lyon, Laboratoire de Physique, UMR 5672, CNRS, \\ École Normale Supérieure de Lyon, 46, allée d'Italie, Lyon cedex 07, F-69364 France \\ E-mail: E.Malek@lmu.de, Henning.Samtleben@ens-lyon.fr
}

ABSTRACT: We use exceptional field theory to establish a duality between certain consistent 7-dimensional truncations with maximal SUSY from IIA to IIB. We use this technique to obtain new consistent truncations of IIB on $S^{3}$ and $H^{p, q}$ and work out the explicit reduction formulas in the internal sector. We also present uplifts for other gaugings of 7 -d maximal SUGRA, including theories with a trombone gauging. Some of the latter can only be obtained by a non-geometric compactification.

Keywords: Flux compactifications, Supergravity Models, Superstring Vacua, String Duality

ArXIV EPRINT: 1510.03433 


\section{Contents}

1 Introduction 1

2 EFT and 7-dimensional maximal gauged SUGRA 3

3 Dualising IIA/IIB truncations $\quad 6$

$\begin{array}{ll}3.1 & \text { Duality as an outer automorphism }\end{array}$

3.2 Example: IIA and IIB on $S^{3}$ and $H^{p, q} \quad 8$

$\begin{array}{lll}3.3 & \text { A no-go theorem on IIA/IIB uplifts } & 12\end{array}$

4 Dualising the 4's $\quad 14$

5 Further examples $\quad \mathbf{1 5}$

$\begin{array}{lll}5.1 & \text { Orbits } 1 \text { and } 2 & 15\end{array}$

$\begin{array}{lll}5.2 & \text { Orbits } 3 \text { and } 4 & 17\end{array}$

$\begin{array}{lll}5.3 & \text { Orbit } 5 & 18\end{array}$

$\begin{array}{llr}5.4 & \text { Orbit } 6 & 19\end{array}$

$\begin{array}{lll}5.5 & \text { Orbits } 7-9 & 19\end{array}$

6 Conclusions 20

\section{Introduction}

The consistent Kaluza-Klein truncation of higher-dimensional (super)gravity to lowerdimensional theories is an old and generically difficult problem due to the highly non-linear gravitational field equations [1]. Typically, consistent truncations require very particular backgrounds together with very particular matter couplings of the higher-dimensional theory, see e.g. [2-4]. Recent progress has come from the realisation of non-toroidal geometric compactifications via generalised Scherk-Schwarz-type compactifications on an extended spacetime within duality covariant formulations of the higher-dimensional supergravity theories [5-12]. In this language, finding consistent Kaluza-Klein reduction Ansätze translates into the search for Scherk-Schwarz twist matrices satisfying a number of differential consistency equations in the physical coordinates. Most recently, this has been used to work out the full Kaluza-Klein reduction for the $\mathrm{AdS}_{5} \times S^{5}$ reduction of IIB supergravity in the framework of exceptional field theory [13].

In this paper we use this framework to study consistent truncations from IIA and IIB supergravity down to seven dimensional gauged supergravities. Specifically, we establish a duality relating consistent IIA and IIB truncations for certain gaugings of maximal 7dimensional supergravity. We then employ this duality to derive new consistent truncations of type IIB theory on the three sphere $S^{3}$, as well as on hyperboloids $H^{p, q}$, which lead to 
compact $\mathrm{SO}(4)$, non-compact $\mathrm{SO}(p, q)$ and non-semisimple $\mathrm{CSO}(p, q, r)$ gaugings, respectively. Finally, we discuss new uplifts to type IIA/IIB of gauged supergravities involving gauging of the trombone scaling symmetry. In this final set of gaugings, we find that some can only be obtained by non-geometric compactifications, ${ }^{1}$ in a set-up reminiscent of that recently discussed in [14].

Let us get more specific about the 7-dimensional theories discussed in this paper. In general, the fluxes in half-maximal supergravity are parametrized by an antisymmetric tensor $X_{A B C}$ of the T-duality group $\mathrm{SO}(d, d)$ [15], which encodes the T-duality chain of [16]

$$
X_{A B C}: H_{a b c} \longrightarrow f_{a b}^{c} \longrightarrow Q_{a}^{b c} \longrightarrow R^{a b c},
$$

as well as two $\mathrm{SO}(d, d)$ vectors $X_{A}$ and $f_{A},[17]$, the latter of which encodes the trombone gaugings. Because the trombone symmetry is an on-shell symmetry, theories with non-zero $f_{A}$ can only be defined at the level of the equations of motion [18]. For $d=3$, i.e. reduction to seven dimensions, $X_{A B C}$ splits into two irreducible representations

$$
\begin{aligned}
\mathbf{2 0} & \longrightarrow \mathbf{1 0}+\mathbf{1 0}^{\prime}, \\
X_{A B C} & =\Gamma_{A B C}{ }^{\alpha \beta} M_{\alpha \beta}+\Gamma_{A B C \alpha \beta} \tilde{M}^{\alpha \beta},
\end{aligned}
$$

with the $\mathrm{SO}(3,3) \Gamma$-matrices (or 't Hooft symbols, see for example appendix B of [19]), and symmetric matrices $M_{\alpha \beta}, \tilde{M}^{\alpha \beta}$. Here the indices $\alpha, \beta=1, \ldots 4$ are fundamental $\mathrm{SL}(4) \simeq \operatorname{Spin}(3,3)$ spinor indices. Similarly, the vectors can be written in terms of the 6 of SL(4) as

$$
X_{A}=\frac{1}{2} \Gamma_{A}^{\alpha \beta} \xi_{\alpha \beta}, \quad f_{A}=\frac{1}{2} \Gamma_{A}^{\alpha \beta} \tau_{\alpha \beta} .
$$

For simplicity's sake we will take $X_{A}=f_{A}=0$ for the following discussion although we will reintroduce them later on.

Depending on the choice of $M_{\alpha \beta}, \tilde{M}^{\alpha \beta}$, there are various one-parameter families of seven-dimensional gaugings most of which are of locally non-geometric origin [19]. A distinguished role is played by the theories satisfying the condition

$$
M_{\alpha \beta} \tilde{M}^{\alpha \beta}=0
$$

First, these can be consistently embedded into the maximal theory and second the subset where either $M_{\alpha \beta}$ or $\tilde{M}^{\alpha \beta}$ is non-degenerate allow for a geometric uplift to the type-I theory in ten dimensions as compactifications on the sphere $S^{3}$ and hyperboloids $H^{p, q}$. For the sphere case, the reduction formulas have been worked out in [4] and later explained in the context of generalized geometry/double field theory $[9,19,20]$. The duality

$$
M_{\alpha \beta} \longleftrightarrow \tilde{M}^{\alpha \beta}
$$

is a symmetry of the quadratic constraints ensuring consistency of the gauging, as a manifestation of a particular triple T-duality [19,21], generated by an element of $O(3,3)$ rather than $\mathrm{SO}(3,3)$.

\footnotetext{
${ }^{1}$ Here we refer to global non-geometry, where the structure group of the manifold is not contained within the geometric subgroup of the U-duality group.
} 
In this paper, we will study the embedding of these structures in the maximal theory with U-duality group SL(5). The above representations are embedded into U-duality representations according to

$$
\begin{aligned}
\mathrm{SO}(3,3) & \subset \mathrm{SL}(5), \\
\mathbf{1 0} & \subset \mathbf{1 5}, \\
\mathbf{1 0}^{\prime} & \subset \mathbf{4 0}^{\prime} .
\end{aligned}
$$

Now the duality (1.5) is no longer a symmetry of one and the same theory. Instead, the different embeddings (1.6) into the representations of the U-duality group induce inequivalent maximal seven-dimensional theories with gauge groups $\operatorname{CSO}(p, q, 1)$ for the IIA background and $\mathrm{SO}(p, q)$ for the IIB background, respectively [22]. These theories only coincide after truncation to the half-maximal sector. The IIA uplift has been given in [11] via a generalized Scherk-Schwarz Ansatz in an exceptional space in the framework of exceptional field theory [23]. Here we realise the duality (1.5) as an outer automorphism of SL(4) acting on the Scherk-Schwarz twist matrices, and thereby derive the full IIB reduction Ansatz. In particular, the duality exchanges the IIA and their dual IIB coordinates within the 10 coordinates of the exceptional space $[24,25]$

$$
10 \longrightarrow 3_{\mathrm{IIA}}+\mathbf{3}_{\mathrm{IIB}}^{\prime}+3+1
$$

We will also show how the triple T-duality acting on the 6's [19]

$$
\xi_{\alpha \beta} \longleftrightarrow \xi^{\alpha \beta}=\frac{1}{2} \epsilon^{\alpha \beta \gamma \delta} \xi_{\gamma \delta}, \quad \tau_{\alpha \beta} \longleftrightarrow \tau^{\alpha \beta}=\frac{1}{2} \epsilon^{\alpha \beta \gamma \delta} \tau_{\gamma \delta},
$$

is realised in the maximal theory.

The paper is organized as follows. In section 2 we briefly review the pertinent structures of the relevant exceptional field theory and its generalized Scherk-Schwarz reduction ansatz. In section 3 we realize the duality (1.5) on the Scherk-Schwarz twist matrix, relating consistent IIA/IIB truncations. As an application we work out the full truncation Ansätze for the internal sectors of the IIA and IIB reductions. In particular, this establishes the consistency of the $S^{3}$ reduction of the IIB theory. Finally, in section 5 we extend the analysis to the construction of more general twist matrices and obtain new uplifts of various maximal supergravities including those in which the trombone scaling symmetry is gauged.

\section{$2 \quad$ EFT and 7-dimensional maximal gauged SUGRA}

Our key tool for the study of consistent truncations is the 'exceptional field theory' (EFT) [23, 26-28] with its associated extended geometry, see [24, 29, 30]. This is the duality covariant formulation of higher-dimensional supergravity which renders manifest the exceptional symmetry groups that are known to appear under dimensional reduction [31]. The formulation of interest for studying reductions to maximal seven-dimensional supergravity, is the SL(5) exceptional field theory. Apart from metric and scalars, it carries 10 
vectors $\mathcal{A}_{\mu}{ }^{a b}$, as well as 5 two-forms $\mathcal{B}_{\mu \nu a}$ and 5 three-forms $\mathcal{C}_{\mu \nu \rho}{ }^{a}$, all fields depending on 7 external and 10 internal coordinates $\left\{x^{\mu}, Y^{a b}\right\}, \mu=0, \ldots, 6 ; a=1, \ldots, 5$ with all fields subject to the section constraint [32]

$$
\partial_{[a b} \otimes \partial_{c d]} \equiv 0
$$

Three-forms enter the Lagrangian only under internal derivatives as $\partial_{a b} \mathcal{C}_{\mu \nu \rho}{ }^{b}$. While the full SL(5) exceptional field theory has not yet been worked out (see [33-35] for EFTs in higher dimensions), its scalar sector has been given and studied in [24, 36, 37]. The 14 scalar fields parametrize a unit-determinant symmetric $5 \times 5$ matrix $\mathcal{M}^{a b}$, i.e. form the coordinates of the coset space $\mathrm{SL}(5) / \mathrm{SO}(5)$. W.r.t. the generalized space, $S L(5)$ generalized diffeomorphisms act according to

$$
\begin{gathered}
\delta V^{a}=\Lambda^{b c} \partial_{b c} V^{a}+2 \partial_{b c} \Lambda^{a b} V^{c}+\frac{2}{5} \partial_{b c} \Lambda^{b c} V^{a}, \\
\delta V_{a}=\Lambda^{b c} \partial_{b c} V_{a}-2 \partial_{a b} \Lambda^{b c} V_{c}-\frac{2}{5} \partial_{b c} \Lambda^{b c} V_{a},
\end{gathered}
$$

on weight zero tensors in the fundamental representations of SL(5). The section constraint (2.1) admits two solutions [25]. Breaking the U-duality group SL(5) down to the geometric $\mathrm{SL}(3)$, the internal coordinates decompose into

$$
Y^{a b} \longrightarrow\left\{Y^{\alpha \beta}, Y^{\alpha 5}\right\} \longrightarrow\left\{Y^{m 4}, Y^{m n}, Y^{m 5}, Y^{45}\right\}, \quad \alpha=1, \ldots, 4 ; \quad m=1,2,3,
$$

cf. (1.7), and it is easy to see that the section constraint (2.1) is satisfied by restricting the coordinate dependence of all fields onto

$$
\begin{cases}y^{m} \equiv Y^{m 4} & \text { (IIA) } \\ \tilde{y}_{m} \equiv \frac{1}{2} \varepsilon_{m n p} Y^{n p} & \text { (IIB) }\end{cases}
$$

respectively. Depending on the higher-dimensional origin, it is convenient to parametrize the scalar matrix $\mathcal{M}^{a b}$ in a IIA or IIB basis according to

$$
\begin{aligned}
\mathcal{M}_{\text {IIA }}^{a b} & =\left(\begin{array}{ccc}
e^{\varphi / 2} g^{2 / 5} g^{m n}+e^{-\varphi / 2} g^{-3 / 5} B^{m} B^{n} & e^{-\varphi / 2} g^{-3 / 5} B^{m} & -g^{2 / 5} g^{m k} e^{\varphi / 2} C_{k}+e^{-\varphi / 2} g^{-3 / 5} C B^{m} \\
e^{-\varphi / 2} g^{-3 / 5} B^{m} & e^{-\varphi / 2} g^{-3 / 5} & e^{-\varphi / 2} g^{-3 / 5} C \\
-g^{2 / 5} g^{m k} e^{\varphi / 2} C_{k}+e^{-\varphi / 2} g^{-3 / 5} C B^{m} & e^{-\varphi / 2} g^{-3 / 5} C & e^{-\varphi / 2} g^{-3 / 5} C^{2}+g^{2 / 5}\left(e^{-\varphi}+e^{\varphi / 2} g^{k l} C_{k} C_{l}\right)
\end{array}\right), \\
\mathcal{M}_{\mathrm{IIB}}^{a b} & =\left(\begin{array}{cc}
g^{-3 / 5} g^{m n} & -g^{-3 / 5} g^{m n} C^{v}{ }_{n} \\
-g^{-3 / 5} g^{n k} C^{u}{ }_{k} & g^{-3 / 5} C^{u}{ }_{m} g^{m n} C^{v}{ }_{n}+g^{2 / 5} H^{u v}
\end{array}\right),
\end{aligned}
$$

where for IIA $g_{m n}$ is the metric, $C_{m}$ is the Ramond-Ramond one-form, $B^{m}=\frac{1}{2} \epsilon^{m n p} B_{n p}$ is the dualised Kalb-Ramond two-form, $C=\frac{1}{3 !} \epsilon^{m n p} C_{m n p}$ is the dualised Ramond-Ramond three-form and $\varphi$ is the dilaton. For IIB, we follow the conventions of [25] so that all fourdimensional indices are placed "upside-down". Thus, $g^{m n}$ represents the metric, $C_{m}{ }^{u}=$ $\left(B_{m}, C_{m}\right)=\frac{1}{2} \epsilon_{m n p}\left(B^{n p}, C^{n p}\right)$ represents the $\mathrm{SL}(2)$ doublet formed from the Kalb-Ramond and Ramond-Ramond two-forms and $H^{u v}$ is the $\mathrm{SL}(2)$ matrix parameterised by the dilaton $\varphi$ and Ramond-Ramond scalar $C_{0}$ as follows

$$
H^{u v}=\left(\begin{array}{cc}
e^{\varphi} & -C_{0} e^{\varphi} \\
-C_{0} e^{\varphi} & e^{\varphi}\left(C_{0}\right)^{2}+e^{-\varphi}
\end{array}\right) .
$$

Throughout the paper the metric will be given in Einstein frame, unless otherwise specified. 
The EFT formulation of supergravity is a powerful tool for the study of consistent truncations, since a number of geometrically non-trivial reductions can be reformulated as generalized Scherk-Schwarz reductions on the extended space [5-11, 19, 38]. In the reduction Ansatz, all dependence on the internal coordinates is carried by an SL(5) valued twist matrix $U_{a} \bar{a}(Y)$ with the scalar fields reducing according to

$$
\mathcal{M}_{a b}(x, Y)=U_{a}{ }^{\bar{a}}(Y) U_{b}{ }^{\bar{b}}(Y) M_{\bar{a} \bar{b}}(x),
$$

and the remaining EFT fields factorizing as [11]

$$
\begin{aligned}
G_{\mu \nu}(x, Y) & =\rho^{-2}(Y) G_{\mu \nu}(x), \\
\mathcal{A}_{\mu}{ }^{a b}(x, Y) & =\rho^{-1}(Y) A_{\mu} \bar{a} \bar{b}(x) U_{\bar{a} \bar{b}}{ }^{a b}(Y), \\
\mathcal{B}_{\mu \nu a}(x, Y) & =\rho^{-2}(Y) B_{\mu \nu \bar{a}}(x) U_{a}{ }^{\bar{a}}(Y), \\
\mathcal{C}_{\mu \nu \rho}{ }^{a}(x, Y) & =\rho^{-3}(Y) C_{\mu \nu \rho}{ }^{a}(x) U_{\bar{a}}{ }^{a}(Y),
\end{aligned}
$$

with a scalar function $\rho(Y)$. The 7-dimensional metric of the full 10-dimensional type II theory, $g_{\mu \nu}$, is related to $G_{\mu \nu}$ above by

$$
g_{\mu \nu}(x, Y)=|g|^{-1 / 5} G_{\mu \nu}(x, Y),
$$

where $|g|$ here is the determinant of the metric in the internal directions and $G_{\mu \nu}(x)$ is the metric of the 7-dimensional gauged SUGRA. Consistency of the reduction Ansatz translates into the set of differential equations [7] (we use the conventions of [11])

$$
\begin{array}{r}
\partial_{a b} U_{(\bar{a}}^{a} U_{\bar{b})}{ }^{b} \stackrel{!}{=}-\rho S_{\bar{a} \bar{b}}, \\
\epsilon^{a b c e f}\left(U_{e f}{ }^{\bar{a} \bar{b}} \partial_{a b} U_{c}{ }^{\bar{c}}-U_{e f} f^{[\bar{a} \bar{b}} \partial_{a b} U_{c}^{\bar{c}]}\right) \stackrel{!}{=} 2 \rho Z^{\bar{a} \bar{b}, \bar{c}}, \\
\partial_{c d} U_{\bar{a} \bar{b}}{ }^{c d}-6 \rho^{-1} U_{\bar{a} \bar{b}}{ }^{c d} \partial_{c d} \rho \stackrel{!}{=}-2 \rho \tau_{\bar{a} \bar{b}},
\end{array}
$$

for the twist matrices, with $U_{a b}{ }^{\bar{a}} \bar{b} \equiv U_{[a}{ }^{\bar{a}} U_{b]}{ }^{\bar{b}}$, and constant tensors $S_{\bar{a} \bar{b}}, Z^{\bar{a} \bar{b}, \bar{c}}, \tau_{\bar{a} \bar{b}}$ transforming in the $\mathbf{1 5}, \mathbf{4 0}$, and $\mathbf{1 0}$, of $\mathrm{SL}(5)$, respectively. These tensors form the torsion of the Weitzenböck connection of EFT [9, 29, 37, 39] and correspond to the embedding tensors of maximal $D=7$ supergravity, which describe the allowed gaugings of the sevendimensional theory [22]. The quadratic constraints which these tensors need to satisfy for consistency are a direct consequence of their definition by (2.10) together with the section constraint (2.1) and ensure that the gauge group closes. For later convenience, we spell out these equations

$$
\begin{aligned}
& S_{\bar{a} \bar{d}} Z^{\bar{d}(\bar{b}, \bar{c})}-\frac{1}{4} \epsilon_{\bar{a} \bar{d} \bar{e} \bar{f} \bar{g}} Z^{\bar{d} \bar{e}, \bar{b}} Z^{\bar{f} \bar{g}, \bar{c}}+\frac{1}{3} \tau_{\bar{a} \bar{d}} Z^{\bar{d}(\bar{b}, \bar{c})}=-\frac{1}{9} \delta_{\bar{a}}^{(\bar{b}} \epsilon^{\bar{c}) \bar{d} \bar{e} \bar{f} \bar{g}} \tau_{\bar{d} \bar{e}} \tau_{\bar{f} \bar{g}}, \\
& S_{\bar{a} \bar{d}} Z^{\bar{b} \bar{c}, \bar{d}}+\frac{1}{6} \epsilon^{\bar{b} \bar{c} \bar{d} \bar{e} \bar{f}} \tau_{\bar{e} \bar{f}} S_{\bar{a} \bar{d}}=-\frac{1}{4} \delta_{\bar{a}}^{[\bar{b}} \epsilon^{\bar{c}] \bar{d} \bar{e} \bar{f} \bar{g}} \tau_{\bar{d} \bar{e}} \tau_{\bar{f} \bar{g}}, \\
& S_{\bar{a} \bar{d}} Z^{\bar{b} \bar{c}, \bar{d}}+\frac{1}{3} \tau_{\bar{a} \bar{d}} Z^{\bar{b} \bar{c}, \bar{d}}=-\frac{2}{9} \delta_{\bar{a}}^{[\bar{b}} \epsilon^{\bar{c}] \bar{d} \bar{e} \bar{f} \bar{g}} \tau_{\bar{d} \bar{e}} \tau_{\bar{f} \bar{g}} .
\end{aligned}
$$

In particular, these identities imply that

$$
W^{\bar{a} \bar{b}, \bar{c}} X_{\bar{a} \bar{b}}=0,
$$


where

$$
W^{\bar{a} \bar{b}, \bar{c}}=-Z^{\bar{a} \bar{b}, \bar{c}}+\frac{1}{3} \epsilon^{\bar{a} \bar{b} \bar{c} \bar{d} \bar{e}} \tau_{\bar{d} \bar{e}},
$$

is the "intertwining tensor" coupling two-forms to the vector field strengths [40] whose rank encodes the number of massive two-forms in the theory. The $X_{\bar{a} \bar{b}}$ are the gauge generators evaluated in the vector representation, which take the form

$$
\left(X_{\bar{a} \bar{b})_{\bar{c}}}{ }^{\bar{d}}=\tau_{\bar{a} \bar{b}, \bar{c}}{ }^{\bar{d}}=\frac{1}{2} \epsilon_{\bar{a} \bar{b} \bar{c} \bar{e} \bar{f}} Z^{\bar{e} \bar{f}, \bar{d}}+2 \delta_{[\bar{a}}^{\bar{d}} S_{\bar{b}] \bar{c}}+\frac{1}{3} \delta_{\bar{c}}^{\bar{d}} \tau_{\bar{a} \bar{b}}+\frac{2}{3} \delta_{[\bar{a}}^{\bar{d}} \tau_{\bar{b}] \bar{c}},\right.
$$

in terms of the embedding tensors (2.10). With $\tau_{\bar{a} \bar{b}}=0$, the corresponding theories are the conventional gaugings of $D=7$ supergravity constructed in [22]. In particular, the gaugings triggered by $S_{\bar{a} \bar{b}}$ correspond to $\operatorname{CSO}(p, q, 5-p-q)$ gauge groups. The corresponding twist matrices for their $D=11$ embedding have been provided in [11]. The gaugings triggered by $Z^{\bar{a} \bar{b}, \bar{c}}$ contain theories with gauge groups $\operatorname{CSO}(p, q, 4-p-q) \times(\mathrm{U}(1))^{4-p-q}$ and IIB origin. We will construct the corresponding twist matrices in this paper. A non-vanishing $\tau_{\bar{a} \bar{b}}$ corresponds to a gauging of the trombone scaling symmetry of the $D=7$ theory, resulting in a theory that can be defined on the level of the equations of motion but does not admit an action [18] while still allowing for an uplift to the IIA/IIB equations of motion.

\section{Dualising IIA/IIB truncations}

In the above we have reviewed how consistent truncations of the IIA/IIB theory are encoded in Scherk-Schwarz twist matrices on the extended space (1.7) satisfying the consistency conditions (2.10) and the section constraint (2.1). In this section, we will first realize the duality (1.5) on the twist matrices and the coordinates of extended space in order to map consistent IIA truncations into consistent IIB truncations. In particular, this will provide the full non-linear reduction Ansätze for the reduction of the IIB theory on $S^{3}$ and the hyperboloids $H^{p, q}$.

At the level of the effective seven-dimensional theories this duality is realized on the embedding tensors that define the maximal gaugings. Decomposing the embedding tensors under the T-duality group as $\mathrm{SL}(5) \longrightarrow \mathrm{SL}(4) \sim \operatorname{Spin}(3,3)$ we find

$$
\begin{aligned}
& 15 \longrightarrow 10 \oplus 4 \oplus \mathbf{1}, \\
& 40^{\prime} \longrightarrow 20^{\prime} \oplus 10^{\prime} \oplus 6 \oplus 4^{\prime} \text {, } \\
& 10 \longrightarrow 6 \oplus 4 \text {. }
\end{aligned}
$$

We will now discuss the $O(3,3)$ transformation $(1.5)$ that exchanges the $\mathbf{1 0} \longleftrightarrow \mathbf{1 0}^{\prime}$ and maps the two 6's into themselves. We will show that it corresponds to a duality between IIA and IIB truncations. This transformation extends $\operatorname{SL}(4) \sim \operatorname{Spin}(3,3)$ to $\operatorname{Pin}(3,3)$, acting on SL(4) as the outer automorphism.

\subsection{Duality as an outer automorphism}

In order to consider type II truncations, we first perform a dimensional reduction of the exceptional space (1.7). In terms of SL(4) irreducible representations, the coordinates 
decompose as $Y^{a b} \longrightarrow\left(Y^{\alpha \beta}, Y^{\alpha 5}\right)$, where $\alpha=1, \ldots 4$, cf. (2.3). We assume no dependence on the $Y^{\alpha 5}$, i.e. reduce the exceptional space to the doubled space of DFT [41, 43-45], see [46]. Depending on the choice of the physical coordinates among the remaining $Y^{\alpha \beta}$, the theory is of IIA or IIB origin according to (2.4). Let us also introduce the notation

$$
Y_{\alpha \beta}=\frac{1}{2} \epsilon_{\alpha \beta \gamma \delta} Y^{\gamma \delta}, \quad \partial^{\alpha \beta}=\frac{1}{2} \epsilon^{\alpha \beta \gamma \delta} \partial_{\gamma \delta},
$$

where $\epsilon^{\alpha \beta \gamma \delta}$ is the 4-dimensional totally-antisymmetric symbol. The flip between IIA and IIB coordinates in (1.7) is then realized as

$$
Y_{\alpha \beta} \longleftrightarrow Y^{\alpha \beta}
$$

We start from the following $G L(4)$ Ansatz for the SL(5) Scherk-Schwarz twist matrix

$$
U_{a}{ }^{\bar{a}}=\left(\begin{array}{cc}
\omega^{-1 / 2} V_{\alpha}{ }^{\bar{\alpha}} & 0 \\
0 & \omega^{2}
\end{array}\right),
$$

with $V_{\alpha}{ }^{\bar{\alpha}} \in \mathrm{SL}(4)$. It follows from (2.10) that this Ansatz can only produce gaugings in the 10's and 6's according to the decomposition of (3.1). The corresponding embedding tensors are given in terms of the twist by

$$
\begin{aligned}
S_{\bar{\alpha} \bar{\beta}} & =M_{\bar{\alpha} \bar{\beta}} \equiv \rho^{-1} \omega V_{(\bar{\alpha}}^{\alpha} \partial_{|\alpha \beta|} V_{\bar{\beta})}{ }^{\beta}, \quad Z^{\overline{5}(\bar{\alpha}, \bar{\beta})}=\tilde{M}^{\bar{\alpha} \bar{\beta}} \equiv \rho^{-1} \omega V_{\alpha}^{(\bar{\alpha}} \partial^{|\alpha \beta|} V_{\beta}^{\bar{\beta})}, \\
2 \tau_{\bar{\alpha} \bar{\beta}} & =-\rho^{-1} \omega\left(\partial_{\alpha \beta} V_{\bar{\alpha} \bar{\beta}}^{\alpha \beta}-5 V_{\bar{\alpha} \bar{\beta}}^{\alpha \beta} \partial_{\alpha \beta} \ln \omega+6 V_{\bar{\alpha} \bar{\beta}}^{\alpha \beta} \partial_{\alpha \beta} \ln \left(\rho^{-1} \omega\right)\right), \\
6 Z^{\overline{5}[\bar{\alpha}, \bar{\beta}]} & =2 \xi^{\bar{\alpha} \bar{\beta}} \equiv \rho^{-1} \omega\left(\partial^{\alpha \beta} V_{\alpha \beta}{ }^{\bar{\alpha} \bar{\beta}}-5 V_{\alpha \beta}{ }^{\bar{\alpha} \bar{\beta}} \partial^{\alpha \beta} \ln \omega\right)
\end{aligned}
$$

where we use $V_{\alpha \beta}{ }^{\bar{\alpha}} \bar{\beta}=V_{[\alpha}{ }^{\bar{\alpha}} V_{\beta]}{ }^{\bar{\beta}}$. The explicit form of these equations shows that combining the flip (3.3) with the $\mathbb{Z}_{2}$ outer automorphism of SL(4)

$$
V_{\alpha}^{\bar{\alpha}} \longleftrightarrow\left(V^{-T}\right)_{\bar{\alpha}}^{\alpha}
$$

induces the duality (1.5) on the embedding tensor. Concretely this takes

$$
M_{\bar{\alpha} \bar{\beta}} \longleftrightarrow \tilde{M}^{\bar{\alpha} \bar{\beta}}, \quad \tau_{\bar{\alpha} \bar{\beta}} \longleftrightarrow \tau^{\bar{\alpha} \bar{\beta}}, \quad \xi^{\bar{\alpha} \bar{\beta}} \longleftrightarrow \xi_{\bar{\alpha} \bar{\beta}},
$$

where $\tau^{\bar{\alpha} \bar{\beta}}=\frac{1}{2} \epsilon^{\bar{\alpha} \bar{\beta} \bar{\gamma} \bar{\delta}} \tau_{\bar{\gamma} \bar{\delta}}$ and $\xi_{\bar{\alpha} \bar{\beta}}=\frac{1}{2} \epsilon_{\bar{\alpha} \bar{\beta} \bar{\gamma} \bar{\delta}} \xi^{\bar{\gamma} \bar{\delta}}$. Additionally, the dualisation of the coordinates (3.3) exchanges IIA and IIB sections so that this duality relates IIA and IIB truncations. The NS-NS sector remains invariant under the duality since in the half-maximal theory both $\mathbf{1 0}$ and $\mathbf{1 0}^{\prime}$ lie in the same $O(3,3)$ orbit [19]. Within the maximal theory the duality (1.5) relates consistent truncations of the maximal theories, which in general have different gauge groups, vacua, and fluctuations.

The gaugings above are the only ones that survive the $\mathbb{Z}_{2}$ projection to the halfmaximal theory [19]. In section 4, we will also discuss gaugings which do not survive the $\mathbb{Z}_{2}$ projection (these are the $\mathbf{2 0}$ ', $\mathbf{4}$ 's and $\mathbf{1}$ ) and we will show that the duality above does not, in general, hold for these cases. 


\subsection{Example: IIA and IIB on $S^{3}$ and $H^{p, q}$}

Before discussing the duality further, let us apply it to work out the consistent truncation of IIB SUGRA on $S^{3}$ and on warped $H^{p, q}$ manifolds. According to the above discussion, these are dual to the consistent truncations of IIA on these manifolds and yield a gauging in the $\mathbf{1 0}^{\prime} \subset \mathbf{4 0 ^ { \prime }}$ with gauge group $\operatorname{CSO}(p, q, r) \times \mathrm{U}(1)^{r}$ where $p+q+r=4$.

Let us begin by reading off $\omega$ and $V_{\alpha}{ }^{\bar{\alpha}}$ from the IIA twist matrices for $\operatorname{CSO}(p, q, r)$ gaugings given in [11]. Here and throughout this paper we will order the rows and columns of $V_{\alpha}^{\bar{\alpha}}$ as $(i, 4, x)$ with $i, j,=1, \ldots, 4-r$ and $x, y=5-r, \ldots, 3$. The twist is

$$
V_{\alpha}{ }^{\bar{\alpha}}=\left(\begin{array}{ccc}
(1-v)^{1 / 4} \delta_{i}{ }^{\bar{\imath}} & -(1-v)^{-1 / 4} \eta_{i j} y^{j} & 0 \\
-(1-v)^{-1 / 4} K \eta^{\bar{\imath}} y_{\bar{\jmath}} & (1-v)^{-3 / 4}(1+K u) & 0 \\
0 & 0 & (1-v)^{1 / 4} \mathbb{I}_{r}
\end{array}\right), \quad \omega=(1-v)^{1 / 10}
$$

with $u=\delta_{i j} y^{i} y^{j}, v=\eta_{i j} y^{i} y^{j}$, and the matrix $\eta_{i j}$ being the $\operatorname{SO}(p-1, q)$ invariant diagonal matrix. Moreover, $y^{i}=Y^{i 4}$ and we make no distinction between un/barred and upper/lower indices on the IIA coordinates $y^{m}$. From (3.5), we further see that setting $\rho=\omega$ induces vanishing trombone parameter $\tau_{\bar{a} \bar{b}}$ as required for these gaugings. Together, the twist matrix then induces the gauging

$$
M_{\bar{\imath} \bar{\jmath}}=\eta_{\bar{\imath} \bar{\jmath}}, \quad M_{\overline{4} \overline{4}}=1, \quad M_{\bar{x} \bar{y}}=0 .
$$

The function $K(u, v)$ appearing in the twist satisfies the differential equation

$$
2(1-v)\left(u \partial_{v} K+v \partial_{u} K\right)=((1+q-p)(1-v)-u) K-1
$$

with $u=\delta_{i j} y^{i} y^{j}$. This can be solved analytically for all allowed values $p, q$. The internal space corresponding to these truncations are warped hyperboloids $H^{p, q}$ together with $r$ flat directions [11].

We now apply the duality (3.3), (3.6), to obtain the IIB truncations on $H^{p, q}$ which give rise to the $\operatorname{CSO}(p, q, r)$ gaugings in the $\mathbf{1 0}^{\prime} \subset \mathbf{4 0 ^ { \prime }}$ such that

$$
\tilde{M}^{\bar{\imath} \bar{\jmath}}=\eta^{\bar{\imath} \bar{\jmath}}, \quad \tilde{M}^{\overline{4} \overline{4}}=1, \quad \tilde{M}^{\bar{x} \bar{y}}=0,
$$

cf. (3.7). The IIB twist matrices are thus

$$
V_{\alpha}{ }^{\bar{\alpha}}=\left(\begin{array}{ccc}
(1-\tilde{v})^{-1 / 4}\left(\delta_{i}{ }^{\bar{\tau}}+K \eta_{i j} \eta^{\bar{\imath}} \tilde{y}^{j} \tilde{y}_{\bar{\jmath}}\right) & (1-\tilde{v})^{1 / 4} K \eta_{i j} \tilde{y}^{j} & 0 \\
(1-\tilde{v})^{1 / 4} \eta^{\bar{\imath}} \tilde{y}_{\bar{\jmath}} & (1-\tilde{v})^{3 / 4} & 0 \\
0 & 0 & (1-\tilde{v})^{-1 / 4} \mathbb{I}_{r}
\end{array}\right), \quad \omega=(1-\tilde{v})^{1 / 10}
$$

with $\rho=\omega$ and where now $\tilde{y}_{i}$ are IIB coordinates (2.4), $\tilde{u}=\delta^{i j} \tilde{y}_{i} \tilde{y}_{j}$ and $\tilde{v}=\eta^{i j} \tilde{y}_{i} \tilde{y}_{j}$. Using (2.7) and the parameterisation (2.5) we can read off the internal space of the compactification. At the origin of the scalar coset, $M_{\bar{a} \bar{b}}(x)=\delta_{\bar{a} \bar{b}}$, we find the background, 
given by:

$$
\begin{aligned}
\stackrel{\circ}{d s}^{2}= & (1+\tilde{u}-\tilde{v})^{-3 / 4}\left[\left(\delta^{i j}+\frac{\eta^{i k} \tilde{y}_{k} \eta^{j l} \tilde{y}_{l}}{1-\tilde{v}}\right) d \tilde{y}_{i} d \tilde{y}_{j}+\delta^{x y} d \tilde{y}_{x} d \tilde{y}_{y}\right] \\
& +(1+\tilde{u}-\tilde{v})^{1 / 4} d s_{7}^{2}, \\
\stackrel{\circ}{B}^{m n}= & (1-\tilde{v})^{-1 / 2}\left(\frac{1}{1+\tilde{u}-\tilde{v}}+K\right) \epsilon^{m n p} \eta_{p q} \tilde{y}^{q}, \\
e^{\dot{\varphi}}= & (1+\tilde{u}-\tilde{v})^{-1 / 2} .
\end{aligned}
$$

Here we let $m, n=1,2,3$ and we denote by $\stackrel{\circ}{ }^{m n}$ the Kalb-Ramond form and by $\stackrel{\circ}{\text { the }}$ dilaton. We recall that following the conventions of [25] and matching the indices of the IIB coordinates (2.4), the four-dimensional IIB indices are "upside-down" compared to the usual placement. The internal space here is the a warped product $H^{p, q} \times \mathbb{R}^{r}$, where $H^{p, q}$ is the surface satisfying $\eta^{i j} \tilde{y}_{i} \tilde{y}_{j}+z^{2}=1$ in $\mathbb{R}^{4-r}$, with $z$ an additional coordinate. This coincides with the IIA background for this truncation, see [11]. The Kalb-Ramond background field strength is given by

$$
\stackrel{\circ}{F}^{m n p}=3 \partial^{[m} \stackrel{\circ}{B}^{n p]}=\frac{\epsilon^{m n p}}{(1-\tilde{v})^{1 / 2}(1+\tilde{u}-\tilde{v})^{2}}(p-q-2+(\tilde{u}-\tilde{v})(p-q)),
$$

upon using (3.10).

Using (2.7), we can furthermore determine the full truncation Ansatz for the internal fields as fluctuations about the background (3.13). To simplify the notation, we will for this discussion not distinguish between barred and un-barred indices and we will simply refer to the IIB coordinates as $y_{i}$, i.e. drop the tilde. Let us start by considering the case where $p+q=4$. The truncation Ansatz can be elegantly formulated in terms of the harmonics

$$
\mathcal{Y}_{\alpha}=\left(y_{m},(1-v)^{-1 / 2}\right), \quad \mathcal{Y}^{\alpha}=\eta^{\alpha \beta} \mathcal{Y}_{\beta},
$$

the auxiliary metric

$$
\tilde{g}^{i j}=\eta^{i j}+\frac{\eta^{i k} y_{k} \eta^{j l} y_{j}}{1-\tilde{v}}, \quad \tilde{g}_{i j}=\eta_{i j}-y_{i} y_{j}
$$

with volume form

$$
\tilde{\omega}^{i j k}=(1-v)^{-1 / 2} \epsilon^{i j k}
$$

and the auxiliary two form

$$
\tilde{B}^{i j}=\tilde{\omega}^{i j k}\left(K \eta_{k l} y^{l}+y_{k}\right) \quad \Longrightarrow \quad 3 \partial^{[i} \tilde{B}^{j k]}=2 \tilde{\omega}^{i j k},
$$

see section 3 of [13] on details of the construction. We note that only for the sphere case, when $\eta_{i j}=\delta_{i j}$, these auxiliary structures coincide with the background (3.13). Furthermore, it will be useful to decompose the scalar fields $M_{a b}(x)$ as

$$
M_{a b}=\left(\begin{array}{cc}
\kappa^{-1}\left(m_{\alpha \beta}+m_{\alpha 5} m_{\beta 5}\right) & \kappa^{3 / 2} m_{\alpha 5} \\
\kappa^{3 / 2} m_{\beta 5} & \kappa^{4}
\end{array}\right),
$$


with SL(4) matrix $m_{\alpha \beta}$. The truncation formulae for the internal components of all IIB fields are then read off from (2.5), (2.7) and yield

$$
\begin{aligned}
g^{i j} & =\kappa^{-5 / 4} \Delta^{3 / 5} \partial^{i} \mathcal{Y}_{\alpha} \partial^{j} \mathcal{Y}_{\beta} m^{\alpha \beta}, \\
H_{u v} & =\Delta^{2 / 5}\left(\begin{array}{cc}
\kappa^{-5 / 2} \mathcal{Y}^{\alpha} \mathcal{Y}^{\beta}\left(m_{\alpha \beta}+m_{\alpha 5} m_{\beta 5}\right) & \mathcal{Y}^{\alpha} m_{\alpha 5} \\
\mathcal{Y}^{\beta} m_{\beta 5} & \kappa^{5 / 2}
\end{array}\right), \\
C^{i j, u} & =\left\{\tilde{B}^{i j}, 0\right\}-\frac{2}{5} \tilde{\omega}^{i j k} \tilde{g}_{k l}\left\{\Delta^{-1} \partial^{l} \Delta,-5 \kappa^{-5 / 2} \Delta^{4 / 5} \partial^{l} \mathcal{Y}^{[\alpha} \mathcal{Y}^{\beta]} \mathcal{Y}^{\gamma} m_{\alpha 5} m_{\beta \gamma}\right\}, \\
g_{\mu \nu} & =\kappa^{3 / 4} \Delta^{-1 / 5} G_{\mu \nu}(x),
\end{aligned}
$$

in terms of the objects (3.15)-(3.19) and with the function $\Delta$ given by

$$
\Delta=\left(\mathcal{Y}^{\alpha} \mathcal{Y}^{\beta} m_{\alpha \beta}\right)^{-5 / 4}
$$

It is straightforward to verify that at the scalar origin $M_{a b}(x)=\delta_{a b}$, these formulae reduce to the background (3.13).

Let us now compare this result to the IIA truncation formulae on the dual background. Define, now in terms of the IIA coordinates, the harmonics

$$
\mathcal{Y}^{\alpha}=\left(y^{i},(1-v)^{-1 / 2}\right), \quad \mathcal{Y}_{\alpha}=\eta_{\alpha \beta} \mathcal{Y}^{\beta},
$$

the auxiliary metric (as before but now with the reverse position of indices)

$$
\tilde{g}_{i j}=\eta_{i j}+\frac{\eta_{i k} y^{k} \eta_{j l} y^{l}}{1-v}, \quad \tilde{g}^{i j}=\eta^{i j}-y^{i} y^{j},
$$

with volume form

$$
\tilde{\omega}_{i j k}=(1-v)^{-1 / 2} \epsilon_{i j k}
$$

and the auxiliary two-form

$$
\tilde{B}_{i j}=\tilde{\omega}_{i j k}\left(K \eta^{k l} y_{l}+y^{k}\right) \quad \Longrightarrow \quad 3 \partial_{[i} \tilde{B}_{j k]}=2 \tilde{\omega}_{i j k}
$$

With the same scalar matrix (3.19), the truncation formulae for the internal components of all IIA fields are again read off from (2.5), (2.7) and yield

$$
\begin{aligned}
g_{i j} & =\kappa^{-5 / 4} \Delta^{3 / 5} \partial_{i} \mathcal{Y}^{\alpha} \partial_{j} \mathcal{Y}^{\beta} m_{\alpha \beta}, \\
e^{\varphi} & =\kappa^{5 / 2} \Delta^{2 / 5}, \\
C_{i} & =\kappa^{-5 / 2} \partial_{i}\left(\mathcal{Y}^{\alpha} m_{\alpha 5}\right), \\
B_{i j} & =\tilde{B}_{i j}-\frac{2}{5} \Delta^{-1} \tilde{\omega}_{i j k} \tilde{g}^{k l} \partial_{l} \Delta, \\
C_{i j k} & =-\kappa^{-3 / 2} \Delta^{4 / 5} \tilde{\omega}_{i j k} m_{\alpha 5} m^{\alpha \beta} \mathcal{Y}_{\beta}, \\
g_{\mu \nu} & =\kappa^{3 / 4} \Delta^{-1 / 5} G_{\mu \nu}(x),
\end{aligned}
$$


in terms of the above objects and with

$$
\Delta=\left(m^{\alpha \beta} \mathcal{Y}_{\alpha} \mathcal{Y}_{\beta}\right)^{-5 / 4}
$$

We can now see that the full reduction formulae of the IIA and IIB truncations coincide for the NS-NS sector and are related by the same SL(4) outer automorphism we have used for the twists, extended to the scalar fields (3.19)

$$
m^{\alpha \beta} \longleftrightarrow m_{\alpha \beta}, \quad \mathcal{Y}_{\alpha} \longleftrightarrow \mathcal{Y}^{\alpha}
$$

Finally, let us also give the reduction formulae when $2 \leq p+q \leq 4$. To keep the notation more compact it will now be useful to use the dualised form potentials. For IIB these are

$$
C_{m}^{u}=\frac{1}{2} \epsilon_{m n p} C^{n p, u}
$$

while for IIA they are

$$
B^{m}=\frac{1}{2} \epsilon^{m n p} B_{n p}, \quad C=\frac{1}{3 !} \epsilon^{m n p} C_{m n p} .
$$

Let us once again start with the IIB reduction. Recall that our convention is that $m=(i, x)$ where $\eta^{x y}=0$ and $\eta^{i j} \neq 0$. Let

$$
\begin{aligned}
\mathcal{Y}_{\alpha} & =\left(y_{i},(1-v)^{1 / 2}, y_{x}\right), & \mathcal{Y}^{\alpha} & =\eta^{\alpha \beta} \mathcal{Y}_{\beta}, \\
\Delta & =\left(m_{\alpha \beta} \mathcal{Y}^{\alpha} \mathcal{Y}^{\beta}\right)^{-5 / 4}, & \tilde{B}_{i} & =\frac{1}{2} \epsilon_{i j k} \tilde{B}^{j k}=(1-v)^{-1 / 4}\left(K \eta_{i j} y^{j}+y_{i}\right),
\end{aligned}
$$

and $\tilde{g}_{i j}, \tilde{g}^{i j}$ as before. Then we obtain the IIB truncation formulae

$$
\begin{aligned}
g^{m n} & =\kappa^{-5 / 4} \Delta^{3 / 5} \partial^{m} \mathcal{Y}_{\alpha} \partial^{n} \mathcal{Y}_{\beta} m^{\alpha \beta}, \\
H_{u v} & =\Delta^{2 / 5}\left(\begin{array}{cc}
\kappa^{-5 / 2} \mathcal{Y}^{\alpha} \mathcal{Y}^{\beta} m_{\alpha \beta} & \mathcal{Y}^{\alpha} m_{\alpha 5} \\
\mathcal{Y}^{\beta} m_{\beta 5} & \kappa^{5 / 2}
\end{array}\right) \\
C_{i}{ }^{u} & =\left\{\tilde{B}_{i}, 0\right\}-\frac{2}{5}(1-v)^{-1 / 2} \tilde{g}_{i j}\left\{\Delta^{-1} \partial^{j} \Delta,-5 \kappa^{-5 / 2} \Delta^{4 / 5} \partial^{j} \mathcal{Y}^{[\alpha} \mathcal{Y}^{\beta]} \mathcal{Y}^{\gamma} m_{\alpha 5} m_{\beta \gamma}\right\}, \\
C_{x}{ }^{u} & =(1-v)^{-1 / 2} \Delta^{4 / 5}\left\{m_{x \alpha} \mathcal{Y}^{\alpha}, \kappa^{-5 / 2}\left(m_{\alpha \beta} m_{x 5}-m_{\alpha 5} m_{x \beta}\right) \mathcal{Y}^{\alpha} \mathcal{Y}^{\beta}\right\}, \\
g_{\mu \nu} & =\kappa^{3 / 4} \Delta^{-1 / 5} G_{\mu \nu}(x) .
\end{aligned}
$$

The corresponding IIA formulae can be given in terms of

$$
\begin{aligned}
\mathcal{Y}^{\alpha} & =\left(y^{i},(1-v)^{1 / 2}, y^{x}\right), & \mathcal{Y}_{\alpha} & =\eta_{\alpha \beta} \mathcal{Y}^{\beta}, \\
\Delta & =\left(m^{\alpha \beta} \mathcal{Y}_{\alpha} \mathcal{Y}_{\beta}\right)^{-5 / 4}, & \tilde{B}^{i} & =(1-v)^{-1 / 4}\left(K \eta^{i j} y_{j}+y^{i}\right) .
\end{aligned}
$$


and read

$$
\begin{aligned}
g_{m n} & =\kappa^{-5 / 4} \Delta^{3 / 5} \partial_{m} \mathcal{Y}^{\alpha} \partial_{n} \mathcal{Y}^{\beta} m_{\alpha \beta}, \\
e^{\varphi} & =\kappa^{5 / 2} \Delta^{2 / 5}, \\
C_{m} & =\kappa^{-5 / 2} \partial_{m}\left(\mathcal{Y}^{\alpha} m_{\alpha 5}\right), \\
B^{i} & =\tilde{B}^{i}-\frac{2}{5}(1-v)^{-1 / 2} \Delta^{-1} \tilde{g}^{k l} \partial_{l} \Delta, \\
B^{x} & =-(1-v)^{-1 / 2} \kappa \Delta^{4 / 5} m^{x \alpha} \mathcal{Y}_{\alpha}, \\
C & =-(1-v)^{-1 / 2} \kappa^{-3 / 2} \Delta^{4 / 5} m_{\alpha 5} m^{\alpha \beta} \mathcal{Y}_{\beta}, \\
g_{\mu \nu} & =\kappa^{3 / 4} \Delta^{-1 / 5} G_{\mu \nu}(x) .
\end{aligned}
$$

\subsection{A no-go theorem on IIA/IIB uplifts}

We have just shown that the IIA truncations with gaugings in the $\mathbf{1 0} \subset \mathbf{1 5}$ induce dual IIB truncations with gaugings in the $\mathbf{1 0}^{\prime} \subset \mathbf{4 0}^{\prime}$, according to the embedding (3.1). A natural question to ask is whether it is possible to obtain the $\mathbf{1 0} \subset \mathbf{1 5}$ gauging by a IIB truncation - or equivalently the $\mathbf{1 0}^{\prime} \subset \mathbf{4 0 ^ { \prime }}$ by a IIA truncation. We will now show that this cannot be done by analysing the symmetries of the embedding tensor.

In order to use symmetry properties of the embedding tensor, we work in the 10dimensional representation with

$$
\tau_{\bar{a} \bar{b}, \bar{c} \bar{d}} \bar{e}^{\bar{f}}=2 \tau_{\bar{a} \bar{b},[\bar{c}}\left[\bar{e} \delta_{\bar{d}]}^{\bar{f}]},\right.
$$

where $\tau_{\bar{a} \bar{b}, \bar{c}} \bar{d}$ represents the embedding tensor as given in (2.14). The consistency equations (2.10) in this representation can conveniently be computed in terms of

$$
E_{\bar{a} \bar{b}}^{a b} \equiv \rho^{-1} U_{[\bar{a}}^{a} U_{\bar{b}]}^{b},
$$

via the generalized Lie derivative

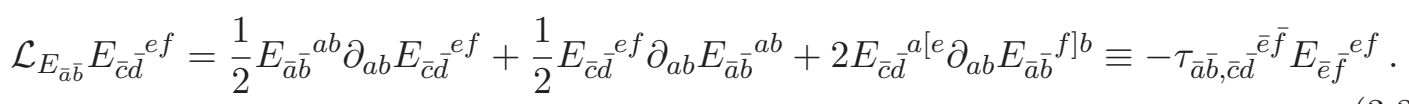

We first assume that the twist only depends on IIA coordinates $Y^{m 4}$ and introduce the following notation

$$
E_{\bar{a} \bar{b}}^{45}=R_{\bar{a} \bar{b}}, \quad E_{\bar{a} \bar{b}}^{m 4}=K_{\bar{a} \bar{b}}^{m}, \quad E_{\bar{a} \bar{b}}^{m 5}=\frac{1}{2} \epsilon^{m n p} L_{n p, \bar{a} \bar{b}}, \quad E_{\bar{a} \bar{b}}^{m n}=\epsilon^{m n p} T_{p, \bar{a} \bar{b}} .
$$

Then the consistency condition (3.37) takes the form

$$
\begin{aligned}
& -\tau_{\bar{a} \bar{b}, \bar{c} \bar{d}} \bar{e} \bar{f} K_{\bar{e} \bar{f}}^{m}=\mathcal{L}_{\bar{a} \bar{b}} K_{\bar{c} \bar{d}}^{m} \equiv K_{\bar{a} \bar{b}}^{n} \partial_{n} K_{\bar{c} \bar{d}}^{m}-K_{\bar{c} \bar{d}}^{n} \partial_{n} K_{\bar{a} \bar{b}}^{m}, \\
& -\tau_{\bar{a} \bar{b}, \bar{c} \bar{d}}^{\bar{e} \bar{f}} R_{\bar{e} \bar{f}}=K_{\bar{a} \bar{b}} m \partial_{m} R_{\bar{c} \bar{d}}-K_{\bar{c} \bar{d}}^{m} \partial_{m} R_{\bar{a} \bar{b}}, \\
& -\tau_{\bar{a} \bar{b}, \bar{c} \bar{d}} \bar{e} \bar{f} L_{m n, \bar{e} \bar{f}}=\mathcal{L}_{\bar{a} \bar{b}} L_{m n, \bar{c} \bar{d}}-3 K_{\bar{a} \bar{b}}^{p} \partial_{[p} L_{m n], \bar{a} \bar{b}}-2 T_{\bar{c} \bar{d}[m} \partial_{n]} R_{\bar{a} \bar{b} \bar{b}}+2 R_{\bar{c} \bar{d}} \partial_{[m} T_{|\bar{a} \bar{b}|, n]}, \\
& -\tau_{\bar{a} \bar{b}, \bar{c} \bar{d}} \overline{\bar{f}} T_{m, \bar{e} \bar{f}}=\mathcal{L}_{\bar{a} \bar{b}} T_{m, \bar{c} \bar{d}}-2 K_{\bar{c} \bar{d}}^{n} \partial_{[n} T_{m], \bar{a} \bar{b}} .
\end{aligned}
$$


Here $\mathcal{L}_{\bar{a} \bar{b}}$ denotes the standard Lie derivative with diffeomorphism parameter $K_{\bar{a} \bar{b}} m$ acting on vectors $K_{\bar{a} \bar{b}} m$ and co-vectors $T_{m, \bar{a} \bar{b}}$ and two-forms $L_{m n, \bar{a} \bar{b}}$. Note that in the first two equations, the right-hand side is antisymmetric under the exchange of $[\bar{a} \bar{b}] \leftrightarrow[\bar{c} \bar{d}]$. Thus, we see that certain contractions of the symmetric part of the embedding tensor vanish. Its symmetric part is given by the intertwining tensor (2.13),

$$
\tau_{\bar{a} \bar{b}, \bar{c} \bar{d}} \bar{e}^{\bar{e} \bar{f}}+\tau_{\bar{c} \bar{d}, \bar{a} \bar{b} \bar{b}}^{\bar{e} \bar{f}}=\epsilon_{\bar{a} \bar{b} \bar{c} \bar{d} \bar{g}} W^{\bar{e} \bar{f}, \bar{g}}=-\epsilon_{\bar{a} \bar{b} \bar{c} \bar{d} \bar{g}} Z^{\bar{e} \bar{f}, \bar{g}}+8 \delta_{\bar{a} \bar{b} \bar{c} \bar{d}}^{\bar{e} \bar{g} \bar{h}} \tau_{\bar{g} \bar{h}}
$$

Thus, a necessary requirement for gaugings to be lifted to IIA is that

$$
3 Z^{\bar{b} \bar{c}, \bar{a}} K_{\bar{b} \bar{c}}^{m}-\epsilon^{\bar{a} \bar{b} \bar{c} \bar{d} \bar{e}} \tau_{\bar{b} \bar{c}} K_{\bar{d} \bar{e}}{ }^{m}=0, \quad 3 Z^{\bar{b} \bar{c}, \bar{a}} R_{\bar{b} \bar{c}}-\epsilon^{\bar{a} \bar{b} \bar{c} \bar{d} \bar{e}} \tau_{\bar{b} \bar{c}} R_{\bar{d} \bar{e}}=0 .
$$

For completeness let us also consider the analogous consistency equations for the IIB theory. In terms of

$$
E_{\bar{a} \bar{b}}^{m n}=\epsilon^{m n p} K_{p, \bar{a} \bar{b}}, \quad E_{\bar{a} \bar{b}}^{45}=\frac{1}{3 !} \epsilon_{m n p} R_{\bar{a} \bar{b}}^{m n p}, \quad E_{\bar{a} \bar{b}}^{m u}=L_{\bar{a} \bar{b}}^{m u},
$$

where $u=4,5$ labels the $\mathrm{SL}(2)$ symmetry of IIB, equation (3.37) becomes

$$
\begin{aligned}
& -\tau_{\bar{a} \bar{b}, \bar{c} \bar{d}} \bar{e} \bar{f} K_{m, \bar{e} \bar{f}}=\mathcal{L}_{\bar{a} \bar{b}} K_{m, \bar{c} \bar{d}}=K_{n, \bar{a} \bar{b}} \partial^{n} K_{m, \bar{c} \bar{c}}-K_{n, \bar{c} \bar{d}} \partial^{n} K_{m, \bar{a} \bar{b}}, \\
& -\tau_{\bar{a} \bar{b}, \bar{c} \bar{d}} \bar{e} \bar{f} R_{\bar{e} \bar{f}} m n p=\mathcal{L}_{\bar{a} \bar{b}} R_{\bar{c} \bar{d}} m n p+6 \epsilon_{u v} L_{\bar{c} \bar{d}}\left[m|u| \partial^{n} L_{\bar{a} \bar{b}} p\right] v, \\
& \left.-\tau_{\bar{a} \bar{b}, \bar{c} \bar{d}} \bar{e}{ }^{\bar{f}} L_{\bar{e} \bar{f}} m u=\mathcal{L}_{\bar{a} \bar{b}} L_{\bar{c} \bar{d}} m u+2 K_{n, \bar{c} \bar{d}} \partial^{[m} L_{\bar{a} \bar{b}} n\right] u .
\end{aligned}
$$

Here $\mathcal{L}_{\bar{a} \bar{b}}$ denotes the standard IIB Lie derivative, i.e. with upside-down indices (see for example [25]), with the diffeomorphism parameter $K_{i, \bar{a} \bar{b}}$. We see that the right-hand side of the first equation is antisymmetric under the exchange of the pair of indices $[\bar{a} \bar{b}] \leftrightarrow[\bar{c} \bar{d}]$. Thus, we find that for a gauging to be of IIB origin, we must have

$$
3 Z^{\bar{b} c \bar{a}} K_{m, \bar{b} \bar{c}}-\epsilon^{\bar{a} \bar{b} \bar{c} \bar{d} \bar{e}} \tau_{\bar{b} \bar{c}} K_{m, \bar{d} \bar{e}}=0 .
$$

Let us now return to the question of whether the $\mathbf{1 0}^{\prime} \subset \mathbf{4 0}^{\prime}$ can come from IIA. To differentiate between the IIA and IIB theories we require dependence on all three internal coordinates and so we consider the case where the gaugings of the $\mathbf{1 0}^{\prime}$ are not degenerate. Using (3.40) it is easy to show that when $\tilde{M}^{\bar{\alpha} \bar{\beta}}=\eta^{\bar{\alpha} \bar{\beta}}$ is not degenerate, (3.41) can only be satisfied by a vanishing twist matrix. Thus these gaugings cannot be obtained from a IIA truncation. In particular, this applies to the $\mathrm{SO}(4)$ theory. By the duality established above, in turn a non-degenerate $M_{\bar{\alpha} \bar{\beta}}=\eta_{\bar{\alpha} \bar{\beta}}$ cannot be obtained from a IIB truncation.

This is interesting in the light of the half-maximal theory, where there is a family of $\mathrm{SO}(4)$ gaugings involving non-degenerate gaugings in both $M_{\bar{\alpha} \bar{\beta}}$ and $\tilde{M}^{\bar{\alpha} \bar{\beta}}$, i.e. in the $\mathbf{1 0}$ and 10' [19]. The result here suggests that such gaugings can only be obtained by violating the section condition, as the corresponding twist matrix would be required to depend both on IIA coordinates and their dual IIB ones. Indeed, this has been shown for the half-maximal theory in $[19,47]$. 


\section{Dualising the 4's}

Recall from (3.1) that the embedding tensor also contains two 4's and one $4^{\prime}$. Can the duality discussed above be extended to these gaugings? Let us begin by relaxing the Ansatz (3.4) in order to have non-zero 4's. Consider first

$$
U_{a}^{\bar{a}}=\left(\begin{array}{cc}
\omega^{-1 / 2} V_{\alpha}^{\bar{\alpha}} & \omega^{-1 / 2} A_{\alpha} \\
0 & \omega^{2}
\end{array}\right) .
$$

The consistency equations are then

$$
\begin{aligned}
& M_{\bar{\alpha} \bar{\beta}}=\rho^{-1} \omega V_{(\bar{\alpha}}^{\alpha} \partial_{|\alpha \beta|} V_{\bar{\beta})}^{\beta}, \quad \tilde{M}^{\bar{\alpha} \bar{\beta}}=\rho^{-1} \omega V_{\alpha}^{(\bar{\alpha}} \partial^{|\alpha \beta|} V_{\beta}{ }^{\bar{\beta})}, \\
& 2 \tau_{\bar{\alpha} \bar{\beta}}=-\rho^{-1} \omega\left(\partial_{\alpha \beta} V_{\bar{\alpha} \bar{\beta}}{ }^{\alpha \beta}-5 V_{\bar{\alpha} \bar{\beta}}^{\alpha \beta} \partial_{\alpha \beta} \ln \omega+6 V_{\bar{\alpha} \bar{\beta}}^{\alpha \beta} \partial_{\alpha \beta} \ln \left(\rho^{-1} \omega\right)\right) \text {, } \\
& 2 \xi^{\bar{\alpha} \bar{\beta}}=\rho^{-1} \omega\left(\partial^{\alpha \beta} V_{\alpha \beta}{ }^{\bar{\alpha} \bar{\beta}}-5 V_{\alpha \beta}{ }^{\bar{\alpha} \bar{\beta}} \partial^{\alpha \beta} \ln \omega\right), \\
& Z^{\bar{\alpha} \overline{5}, \overline{5}}=\rho^{-1} \omega V_{\alpha \beta} \bar{\alpha} \bar{\beta} \partial^{\alpha \beta} A_{\bar{\beta}}+\left(\tilde{M}^{\bar{\alpha} \bar{\beta}}+\xi^{\bar{\alpha} \bar{\beta}}\right) A_{\bar{\beta}},
\end{aligned}
$$

where $A_{\bar{\alpha}}=V_{\bar{\alpha}}{ }^{\alpha} A_{\alpha}$. We see that the equations for the 10's and 6's are unchanged but additionally the $\mathbf{4}^{\prime} \subset \mathbf{4 0}^{\prime}$ can be gauged. If we instead take the Ansatz

$$
U_{a}^{\bar{a}}=\left(\begin{array}{cc}
\omega^{-1 / 2} V_{\alpha}^{\bar{\alpha}} & 0 \\
\omega^{2} B^{\bar{\alpha}} & \omega^{2}
\end{array}\right),
$$

we again find the same 10's and 6's as in (3.5) but additionally the following can be gauged:

$$
\begin{aligned}
& \tau_{\bar{\alpha} \overline{5}}=-\frac{1}{2} \rho^{-1} \omega V_{\bar{\alpha} \bar{\beta}}^{\alpha \beta} \partial_{\alpha \beta} B^{\bar{\beta}}-B^{\bar{\beta}} \tau_{\bar{\alpha} \bar{\beta}}, \\
& Z^{\bar{\alpha} \bar{\beta}, \bar{\gamma}}=\frac{1}{2} \rho^{-1} \omega \epsilon^{\bar{\alpha} \bar{\beta} \bar{\delta} \bar{\rho}} V_{\bar{\delta} \bar{\rho}}^{\delta \rho} \partial_{\delta \rho} B^{\bar{\gamma}}-\frac{2}{3} \epsilon^{\bar{\alpha} \bar{\beta} \bar{\gamma} \bar{\rho}}\left(S_{\bar{\rho} \overline{5}}+B^{\bar{\delta}} M_{\bar{\rho} \bar{\delta}}\right)+2 B^{[\bar{\alpha}}\left(\tilde{M}^{\bar{\beta}] \bar{\gamma}}+\xi^{\bar{\beta}] \bar{\gamma}}\right)-2 B^{[\bar{\alpha}} \xi^{\bar{\beta} \bar{\gamma}]}, \\
& S_{\bar{\alpha} \overline{5}}=-\frac{1}{2} \rho^{-1} \omega V_{\bar{\alpha} \bar{\beta}}^{\alpha \beta} \partial_{\alpha \beta} B^{\bar{\beta}}-B^{\bar{\beta}} M_{\bar{\alpha} \bar{\beta}}, \\
& S_{\overline{5} \overline{5}}=-B^{\bar{\alpha}}\left(2 S_{\bar{\alpha} \overline{5}}+B^{\bar{\beta}} M_{\bar{\alpha} \bar{\beta}}\right) \text {. }
\end{aligned}
$$

The SL(4) (co-)vectors $A_{\alpha}$ and $B^{\alpha}$ should be exchanged by the outer automorphism of SL(4) so that

$$
V_{\alpha}^{\bar{\alpha}} \longleftrightarrow\left(V_{\bar{\alpha}}^{\alpha}\right)^{-T}, \quad \partial_{\alpha \beta} \longleftrightarrow \partial^{\alpha \beta}, \quad A_{\alpha} \longleftrightarrow B^{\alpha} .
$$

This maps a solution of the equations (4.4) to a solution of (4.2) but not vice versa. Thus, it is not in general possible to map a twist that gauges the $\mathbf{4}^{\prime} \subset \mathbf{4 0}^{\prime}$ into a twist gauging the $\mathbf{4} \subset \mathbf{1 5}, \mathbf{4} \subset \mathbf{1 0}$ and $\mathbf{2 0}^{\prime} \subset \mathbf{4 0 ^ { \prime }}$. Furthermore, if we start with a gauging of the $\mathbf{4}^{\prime} \subset \mathbf{4 0 ^ { \prime }}$ that satisfies the quadratic constraints $(2.11)$ and perform the duality to obtain a gauging in the $\mathbf{4} \subset \mathbf{1 5}, \mathbf{4} \subset \mathbf{1 0}$ and $\mathbf{2 0}^{\prime} \subset \mathbf{4 0}$, then this dual gauging does not in general satisfy the quadratic constraint. Then the dual gaugings do not define a consistent gauged SUGRA. We will see an example of this in section 5.2. 


\begin{tabular}{|c|c|c|c|c|c|}
\hline Orbit & $M_{\bar{\alpha} \bar{\beta}}$ & $\tilde{M}^{\bar{\alpha} \bar{\beta}}$ & $Z^{\bar{\alpha} \overline{5}, \overline{5}}$ & $\xi^{\overline{2} \overline{3}}$ & $\tau_{\overline{1} \overline{4}}$ \\
\hline 1 & $\operatorname{diag}(\eta, 1,0,0)$ & $\operatorname{diag}(0,0, \eta, 1)$ & 0 & 0 & 0 \\
2 & $\operatorname{diag}(1,0,0,0) \cos \alpha$ & $\operatorname{diag}(0,0,0,1) \sin \alpha$ & 0 & 0 & 0 \\
3 & $\operatorname{diag}\left(\eta, \eta^{\prime}, 1,0\right)$ & 0 & $(0,0,0,1)$ & 0 & \\
4 & $\operatorname{diag}(\eta, 1,0,0)$ & 0 & $(0,0,0,1)$ & 0 & 0 \\
5 & $\operatorname{diag}(1,0,0,0) \cos \alpha$ & $\operatorname{diag}(0,0,0,1) \sin \alpha$ & $(0,0,1,0)$ & 0 & 0 \\
6 & $\operatorname{diag}(0,0,0,0)$ & $\operatorname{diag}(0,0,0,0)$ & 0 & $\lambda-1$ & $\lambda$ \\
7 & $\operatorname{diag}(\eta, 1,0,0)$ & 0 & 0 & $-a$ & $a$ \\
8 & $\operatorname{diag}(\eta, 1,0,0)$ & $\operatorname{diag}(0,0, \eta, 1)$ & 0 & $-a$ & $a$ \\
9 & $\operatorname{diag}(1,0,0,0) \cos \alpha$ & $\operatorname{diag}(0,0,0,1) \sin \alpha$ & 0 & $-a$ & $a$ \\
\hline
\end{tabular}

Table 1. Orbits of gaugings for which we will construct uplifts. Each $\alpha$ in the range $-\pi / 2 \leq \alpha \leq \pi$, each $\lambda=1, \frac{1}{2}, 0$, each $\eta, \eta^{\prime}= \pm 1$ and each $a \in \mathbb{R}$ labels different inequivalent orbits.

\section{$5 \quad$ Further examples}

We will now use our twist Ansätze (3.4), (4.1) and (4.3) and the duality discussed above to obtain new uplifts of various maximal gauged SUGRAs. This is not an exhaustive list of solutions to the quadratic constraints, but rather a selection of examples for which uplifts to type II SUGRA can be constructed nicely with the twist Ansätze we have considered so far. The gaugings we consider are summarised in table 1. Each value of $\alpha$ in the range $-\pi / 2 \leq \alpha \leq \pi$, as well as each $\lambda$ taking the values $\lambda=1, \frac{1}{2}, 0$, each $\eta, \eta^{\prime}= \pm 1$ and each $a \in \mathbb{R}$ labels different inequivalent orbits. Note that for orbits 1 and 7-9 we have indicated that the gaugings in the $\mathbf{4}$ vanish. This is because any non-zero gaugings in the $\mathbf{4}$ allowed by the quadratic constraint (2.11) can be removed by an SL(5) transformation and thus lead to equivalent 7 -dimensional theories. Orbits 6-9 involve the trombone gauging (when $\lambda \neq 0$ ) and thus the 7-dimensional theories they represent do not admit an action principle. We will see in section 5.5 that in some cases their uplifts are non-geometric, where the trombone scaling symmetry is used to patch together the solution.

\subsection{Orbits 1 and 2}

In section 3.3 we showed that non-degenerate gaugings in the $\mathbf{1 0}$ descend from IIA and those in the $\mathbf{1 0}^{\prime}$ descend from IIB. Let us now uplift gaugings which mix the $\mathbf{1 0}$ and $\mathbf{1 0}^{\prime}$. The quadratic constraint is now

$$
\tilde{M}^{\bar{\alpha} \bar{\beta}} M_{\bar{\alpha} \bar{\gamma}}=0 .
$$

The solutions are given by orbits $4-11$ of [19].

Orbit 1. This orbit can be represented by the gaugings

$$
M_{\bar{\alpha} \bar{\beta}}=\operatorname{diag}(\eta, 1,0,0), \quad \tilde{M}^{\bar{\alpha} \bar{\beta}}=\operatorname{diag}(0,0, \eta, 1) .
$$


These correspond to an embedding of orbits 6 and 9 (with $\alpha=\pi / 4$ ) of [19] into the maximal theory.

The twist matrices are given by

$$
\begin{aligned}
V_{\alpha}^{\bar{\alpha}} & =\left(\begin{array}{cccc}
(1-v)^{1 / 4} & -\eta y_{1}(1-v)^{-1 / 4} & 0 & 0 \\
y_{1}(1-v)^{-1 / 4} & (1-v)^{1 / 4} & 0 & 0 \\
0 & 0 & (1-v)^{3 / 4} & -y_{1}(1-v)^{1 / 4} \\
0 & 0 & (1-v)^{1 / 4} \eta y_{1} & (1-v)^{3 / 4}
\end{array}\right), \\
\omega & =(1-v)^{1 / 10},
\end{aligned}
$$

with $\rho=\omega$ and where $v=\eta y_{1}^{2}$ and $u=y_{1}^{2}$. From (2.5) we find the internal space in string frame to be

$$
\begin{aligned}
& \stackrel{\circ}{d s}^{2}=(1-v)^{-1} d y_{1}^{2}+d y_{2}^{2}+d y_{3}^{2}-2 y_{1}(\eta-1)(1-v)^{1 / 2}(1+u-v)^{-1} d y_{2} d y_{3}+d s_{7}^{2}, \\
& \stackrel{\circ}{B}_{23}=y_{1}(\eta-1)(1-v)^{1 / 2}(1+u-v)^{-1}, \quad e^{\varphi}=(1+u-v)^{-1 / 2} .
\end{aligned}
$$

Note that when $\eta=1$ the background is the Kaluza-Klein circle encountered in (3.13). However, the internal space will be different at other points in the scalar moduli space. It is of course also possible to generate the gaugings

$$
M_{\bar{\alpha} \bar{\beta}}=\operatorname{diag}(0,0, \eta, 1), \quad \tilde{M}^{\bar{\alpha} \bar{\beta}}=\operatorname{diag}(\eta, 1,0,0),
$$

by applying the duality discussed in section 3.1. As before, the internal space remains the same under the duality.

Orbit 2. These orbits describe an embedding of orbits 11 of [19] into the maximal theory. The gaugings are

$$
M_{\bar{\alpha} \bar{\beta}}=\operatorname{diag}(0,1,0,0) \cos \alpha, \quad \tilde{M}^{\bar{\alpha} \bar{\beta}}=\operatorname{diag}(0,0,0,1) \sin \alpha,
$$

where $-\pi / 2 \leq \alpha \leq \pi$ gives the range of inequivalent orbits. The twist matrices are given by

$$
V_{\alpha}^{\bar{\alpha}}=\left(\begin{array}{cccc}
1-y_{1} \cos \alpha & 0 & 0 \\
0 & 1 & 0 & 0 \\
0 & 0 & 1 & -y_{1} \sin \alpha \\
0 & 0 & 0 & 1
\end{array}\right), \quad \omega=1,
$$

where $y_{1}=Y^{14}$ and the internal space is given by

$$
\begin{aligned}
& d s^{2}=d y_{1}^{2}+d y_{2}^{2}+\left(d y_{3}-y_{1} \sin \alpha d y_{2}\right)^{2}+d s_{7}^{2}, \\
& B_{23}=y_{1} \cos \alpha,
\end{aligned}
$$

with all other fields vanishing. The dual gaugings $M_{\bar{\alpha} \bar{\beta}} \longleftrightarrow \tilde{M}^{\bar{\alpha} \bar{\beta}}$ are in this case equivalent to the gaugings discussed. 


\subsection{Orbits 3 and 4}

When $M_{\bar{\alpha} \bar{\beta}}$ and $Z^{\bar{\alpha} \overline{5}, \overline{5}}$ are the only non-zero gaugings, the quadratic constraint is

$$
M_{\bar{\alpha} \bar{\beta}} Z^{\bar{\beta} \overline{5}, \overline{5}}=0 .
$$

Thus, $Z^{\bar{\alpha} \overline{5}, \overline{5}} \neq 0$ only when $M_{\bar{\alpha} \bar{\beta}}$ is degenerate. Let us consider separately the cases where $M_{\bar{\alpha} \bar{\beta}}$ has rank 3 and rank 2, corresponding to orbits 3 and 4 in table 1, respectively.

Orbit 3. Take $M_{\bar{\imath} \bar{\jmath}}=\eta_{\bar{\imath} \bar{\jmath}}, M_{\overline{4} \overline{4}}=1$ and $M_{\overline{3} \overline{3}}=0$, with $\bar{\imath}, \bar{\jmath}=1,2$ and all other elements vanishing. Then by (5.9) we can only have

$$
Z^{\overline{3} \overline{5}, \overline{5}}=c .
$$

We could use an SL(5) transformation to set $c=1$ but we will not do so here to keep track of $c$ in the internal space. However, the reader should keep in mind that all values of $c \neq 0$ correspond to equivalent 7-dimensional theories.

From the no-go theorem (3.41) one finds that this gauging cannot be obtained by a IIA truncation. It can, however, be lifted to 10-dimensional IIB SUGRA using the Ansatz (4.1) with the same $V_{\alpha}^{\bar{\alpha}}$ as in (3.12) with $r=1$ and with

$$
A_{4}=-c y_{3}(1-v)^{-1 / 4}, \quad A_{i}=A_{3}=0
$$

where $y_{3}=Y^{12}$ is the third IIB coordinate. Recall that the other two coordinate are given by $y_{1}=Y^{14}, y_{2}=Y^{24}$. The background for this truncation is given by

$$
\begin{aligned}
\stackrel{\circ}{d s}^{2}= & d s_{7}^{2}+(1-v)^{-1}\left[\delta^{i j} d y_{i} d y_{j}-\frac{\left(\eta^{i j} y_{i} d y_{j}\right)^{2}}{1+u-v}\right] \\
& +(1+u-v)\left[d y_{3}+(1-v)^{-1 / 2} \frac{1+K(1+u-v)}{1+u-v} \eta^{i j} y_{i} d y_{j}\right]^{2}, \\
\check{C}^{i j}= & -c y_{3}(1-v)^{-1 / 2} \epsilon^{i j} .
\end{aligned}
$$

As before, we use the convention of [25] where IIB indices are placed "upside-down" and $\stackrel{C}{ }^{i j}$ labels the Ramond-Ramond two-form. The metric here is the T-dual of the $H^{p, q}$ solutions in (3.13). Furthermore, only the two-form depends on $c$ and the NS-NS sector remains invariant as $c$ is turned on.

Orbit 4. Take $M_{\overline{1} \overline{1}}=\eta, M_{\overline{4} \overline{4}}=1$ and all other components vanishing. Then by (5.9) we can have the gaugings

$$
Z^{\overline{2} \overline{5}, \overline{5}}=c, \quad Z^{\overline{3} \overline{5}, \overline{5}}=d .
$$

One can use an SL(5) transformation to set $c=0$ and $d=1$ but we will not do so here to keep track of where the gaugings appear in the internal space. Once again, however, the reader should remember that different values of $c$ and $d$ (with at least one non-vanishing) correspond to the same 7-dimensional theory. 
We again use the Ansatz (4.1) with $V_{\alpha}^{\bar{\alpha}}$ as in (3.12) with $r=2$ and solve the gauging of the $Z^{\bar{\alpha} \overline{5}, \overline{5}}$ by

$$
A_{1}=(1-v)^{-1 / 4}\left(c y_{3}-d y_{2}\right),
$$

with all other $A_{\alpha}=0, \alpha \neq 1$. The twist now only depends on $y_{1}=Y^{14}, y_{2}=Y^{24}$ and $y_{3}=Y^{34}$ and so gives an uplift to IIA supergravity. From (2.5) the internal space is found to be

$$
\begin{aligned}
\stackrel{\stackrel{\circ}{d}_{11}^{2}=}{ } & (1+u-v)^{-2 / 3}\left[d y_{2}^{2}+d y_{3}^{2}+\left(d z+C_{1} d y_{1}\right)^{2}\right] \\
& +(1+u-v)^{1 / 3} \frac{d y_{1}^{2}}{1-v}+(1+u-v)^{-2 / 3} d s_{7}^{2}, \\
\stackrel{\circ}{C}_{23 z}= & (1+u-v)^{-1}(1-v)^{1 / 2}(1-\eta) y_{1}, \\
\stackrel{C}{C}_{1}= & (1-v)^{-1 / 2}\left(c y_{3}-d y_{2}\right) .
\end{aligned}
$$

This is the same circle/hyperbola reduction as in (3.13) but with an additional RamondRamond one-form $\stackrel{\circ}{C}_{1}$ turned on. Similar to orbit 3, only the Ramond-Ramond one-form depends on $c$ and $d$.

To conclude the discussion of these orbits, let us consider the dual gaugings. The duality would give gaugings of the $\mathbf{4} \subset \mathbf{1 5}, \mathbf{4} \subset \mathbf{1 0}$ with

$$
S_{\bar{\alpha} 5}=\tau_{\bar{\alpha} 5},
$$

as well as possibly the $\mathbf{2 0}^{\prime}$. However, these gaugings violate the quadratic constraint (2.11) and hence they do not define a consistent gauged SUGRA.

\subsection{Orbit 5}

For the gaugings $M_{\bar{\alpha} \bar{\beta}}=\operatorname{diag}(0,1,0,0) \cos \alpha$ and $\tilde{M}^{\bar{\alpha} \bar{\beta}}=\operatorname{diag}(0,0,0,1) \sin \alpha$ the quadratic constraint allows the 4 's

$$
Z^{\bar{\alpha} \overline{5}, \overline{5}}=(d, 0, c, e) .
$$

We can use an SL(5) transformation to make two of these vanish and scale the third. Let us thus take $d=e=0$ but keep $c \neq 1$ in general so that we can see where it ends up in the internal space. The twist matrix is then given by Ansatz (4.1) with $V_{\alpha}{ }^{\bar{\alpha}}$ as in (5.7) and

$$
A_{\overline{3}}=c y_{1} \text {. }
$$

The internal space is then given by

$$
\begin{aligned}
\stackrel{\circ}{d s}^{2} & =d y_{1}^{2}+d y_{2}^{2}+\left(d y_{3}-y_{1} \sin \alpha d y_{2}\right)^{2}+d s_{7}^{2}, \\
\stackrel{\circ}{B}_{23} & =y_{1} \cos \alpha, \\
\stackrel{\circ}{C}_{2} & =-c y_{1}^{2} \sin \alpha, \\
\stackrel{\circ}{C}_{3} & =c y_{1} .
\end{aligned}
$$

As for orbits 3 and 4 we find that the parameter $c$ only appears in the Ramond-Ramond 1-form. The dual gaugings would again not satisfy the quadratic constraint (2.11). 


\subsection{Orbit 6}

To keep our formulae simple we will actually uplift the gaugings

$$
\tau_{\overline{1} \overline{4}}=3(\lambda-1), \quad \xi^{\overline{2} \overline{3}}=3 \lambda,
$$

with inequivalent gaugings for $\lambda=1, \frac{1}{2}, 0$. We can obtain these gaugings easily using the block-diagonal Ansatz for the twist matrix (3.4) and by choosing the scalars $\rho$ and $\omega$ appropriately.

The twist matrix is given by $V_{\alpha}{ }^{\bar{\alpha}}=\delta_{\alpha}{ }^{\bar{\alpha}}$ with scalars $\omega=\left(1-y_{1}\right)^{6 \lambda / 5}$ and $\rho=$ $(1-a \cdot y)^{6 \lambda / 5-1}$. The internal space in string frame is

$$
\stackrel{\dot{d}_{s}}{2}=d y_{m} d y^{m}+(1-a \cdot y)^{2} d s_{7}^{2}, \quad e^{\dot{\varphi}}=(1-a \cdot y)^{3 \lambda} .
$$

We can see that the string-frame metric is independent of $\lambda$ and the dilaton tunes between the different gaugings. In particular, when $\lambda=1$ we have a standard 7-dimensional gauged SUGRA, whereas for the cases $\lambda=0$ and $\lambda=1 / 2$ the 7 -dimensional theory does not have an action principle, even though it can still be uplifted to 10-dimensional SUGRA. For each $\lambda$ the outer automorphism discussed in section 3.1 relates equivalent gaugings.

\subsection{Orbits $7-9$}

The gaugings we consider here involve some of the gaugings encountered previously in this paper together with both 6's. These can be uplifted by using almost the same twist matrices as without the 6's. In particular we will keep $V_{\alpha}{ }^{\bar{\alpha}}$ unchanged but change $\rho=\omega$. Let us write $\rho=\omega=\omega_{0} h$, where $\omega_{0}$ is the value of $\omega$ where the 6's vanish. The function $h$ then has to satisfy

$$
2 \tau_{\bar{\alpha} \bar{\beta}}=-2 \xi_{\bar{\alpha} \bar{\beta}}=5 V_{\bar{\alpha} \bar{\beta}}^{\alpha \beta} \partial_{\alpha \beta} \ln h .
$$

Orbit 7. Let us start with the IIA sphere/hyperboloid case (3.8) where $\omega_{0}=(1-v)^{1 / 10}$. The quadratic constraint (2.11) implies the only gaugings with non-zero $\xi^{\bar{\alpha} \bar{\beta}}=-\tau^{\bar{\alpha} \bar{\beta}}$ are given by

$$
M_{\bar{\alpha} \bar{\beta}}=\operatorname{diag}(\eta, 1,0,0), \quad \tau_{\overline{1} \overline{4}}=-\xi^{\overline{2} \overline{3}}=a .
$$

For $a=0$ these are $S^{1}$ and $H^{1}$ reductions. Now, we find

$$
h=\exp \left(\frac{2 a \arcsin \left(\sqrt{\eta} y_{1}\right)}{5 \sqrt{\eta}}\right) .
$$

The internal space in string-frame is given by

$$
\begin{aligned}
\stackrel{\circ}{d s}^{2} & =\frac{1}{1-v} d y_{1}^{2}+\frac{1}{1+u-v}\left(d y_{2}^{2}+d y_{3}^{2}\right)+d s_{7}^{2}, \\
\stackrel{\circ}{B}_{23} & =(1-v)^{1 / 2}(1+u-v)(\eta-1) y_{1}, \\
e^{\varphi} & =(1+u-v)^{-1 / 2} \exp \left(\frac{a \arcsin \left(\sqrt{\eta} y_{1}\right)}{\sqrt{\eta}}\right) .
\end{aligned}
$$


We see that when $\eta=1$, the internal space is non-geometric because the dilaton is not globally well-defined. Instead, it is patched by the trombone scaling symmetry of the equations of motion. This is a reminiscent of the non-geometric construction in [14] albeit in seven dimensions.

Orbit 8. For the gaugings

$$
M_{\bar{\alpha} \bar{\beta}}=\operatorname{diag}(\eta, 1,0,0), \quad \tilde{M}^{\bar{\alpha} \bar{\beta}}=\operatorname{diag}(0,0, \eta, 1), \quad \tau_{1 \overline{4}}=-\xi^{\overline{2} \overline{3}}=a,
$$

with $V_{\alpha}{ }^{\bar{\alpha}}$ and $\omega$ as in (5.3), the consistency condition on $h,(5.22)$, has the same solution $h$ as in (5.24). We find the internal space in string-frame

$$
\begin{aligned}
\stackrel{\circ}{d}^{2} & =(1-v)^{-1} d y_{1}^{2}+d y_{2}^{2}+d y_{3}^{2}+2 y(\eta-1)(1-v)^{1 / 2}(1+u-v)^{-1} d y_{2} d y_{3}+d s_{7}^{2}, \\
\stackrel{\circ}{B}_{23} & =(1-v)^{1 / 2}(1+u-v)^{-1} y(\eta-1), \\
e^{\dot{\varphi}} & =(1+u-v)^{-1 / 2} \exp \left(\frac{a \arcsin \left(\sqrt{\eta} y_{1}\right)}{\sqrt{\eta}}\right) .
\end{aligned}
$$

Orbit 9. For the gaugings

$$
M_{\bar{\alpha} \bar{\beta}}=\operatorname{diag}(0,1,0,0) \cos \alpha, \quad \tilde{M}^{\bar{\alpha} \bar{\beta}}=\operatorname{diag}(0,0,0,1) \sin \alpha, \quad \tau_{\overline{1} \overline{4}}=-\xi^{\overline{2} \overline{3}}=a,
$$

with $V_{\alpha}^{\bar{\alpha}}$ and $\omega_{0}$ as in (5.7) we find

$$
h=\exp \left(\frac{2 a}{5} y_{1}\right)
$$

The internal space in string-frame is

$$
\begin{aligned}
\stackrel{\circ}{d s}^{2} & =d y_{1}^{2}+d y_{2}^{2}+\left(d y_{3}-y_{1} \sin \alpha d y_{2}\right)^{2}+d s_{7}^{2}, \\
\stackrel{\circ}{B}_{23} & =y_{1} \cos \alpha, \\
e^{\dot{\varphi}} & =\exp \left(a y_{1}\right) .
\end{aligned}
$$

\section{Conclusions}

In this paper we studied consistent truncations of type IIA and IIB SUGRA to 7dimensional maximal gauged SUGRA using exceptional field theory. By using a $G L(4)$ Ansatz for the twist matrices, we showed that IIA/IIB consistent truncations are related by the outer automorphism of SL(4) which acts on the irreducible representations of the embedding tensor as

$$
\mathbf{1 0} \longleftrightarrow \mathbf{1 0}^{\prime}, \quad \mathbf{6}_{40} \longleftrightarrow \mathbf{6}_{40}, \quad \mathbf{6}_{10} \longleftrightarrow \mathbf{6}_{10} .
$$

Here $\mathbf{6}_{40}$ and $\mathbf{6}_{10}$ denote the $\mathbf{6}^{\prime}$ 's coming from the $\mathbf{4 0}^{\prime}$ of SL(5) and from the trombone gauging, respectively. We also showed that this duality between IIA and IIB consistent truncations always exists when the embedding tensor has vanishing components in the 
$4^{\prime}$ of SL(4). Otherwise, the dual gaugings will in general not satisfy the quadratic constraints (2.11).

We used this duality to prove the consistent truncation of IIB on $S^{3}$ and $H^{p, q}$ by constructing twist matrices that give rise to the relevant $\mathrm{CSO}(p, q, r)$ gaugings with embedding tensor in the $\mathbf{4 0}^{\prime}$. The twist matrices are dual to those describing the IIA uplift of gaugings in the $\mathbf{1 5}$ [11]. Using the dictionary between EFT and IIA/IIB fields, we used the twist matrices to derive the full truncation Ansätze for the internal sectors of the IIA and IIB reductions. They were shown to coincide in the NS-NS sector. This is a general feature of the duality: it relates truncations with the same NS-NS sector. Finally, from the form of the consistency equations we derived some no-go theorems showing that non-degenerate gaugings with IIA origin cannot also be uplifted to IIB and vice versa.

In the second part of this paper we further generalised the twist matrices of [11] to uplift other gaugings of 7-dimensional maximal gauged SUGRA to type II SUGRA. These examples include gaugings of the $\mathbf{1 5}$ and $\mathbf{4 0}$ simultaneously, and of the trombone, where the gauged SUGRA does not admit a Lagrangian. In the latter case, the internal space of the truncation is only well-defined up to the $\mathbb{R}^{+}$scaling symmetry of the equations of motion. Among the direct applications of these uplift formulas is the higher-dimensional embedding of the vacua found in the lower-dimensional theories, such as [48].

The twist matrices used throughout this paper are defined in local patches. For the truncation to be consistent, these twist matrices must yield a generalised parallelisation [9]. To show this we would have to patch our twist matrices to obtain globally well-defined vector fields. A patching prescription for exceptional field theory is still lacking, although it is known for double field theory [49-52]. Whatever this covariant patching prescription will be, it should consist of the global SL(5) $\times \mathbb{R}^{+}$symmetries of the 7 -dimensional SUGRA. We can thus argue that our twist matrices are well-defined by checking that the internal space they define is well-defined up to $\mathrm{SL}(5) \times \mathbb{R}^{+}$dualities. This is indeed the case for all the examples given here.

The duality established in this paper exchanges IIA and IIB consistent truncations, by relating different irreducible representations of the embedding tensor of 7-dimensional gauged supergravity according to the embedding (3.1). Similar dualities are expected to arise in all dimensions. In contrast to the 7-dimensional case, for all other dimensions the embedding tensor $X_{A B C}$ of the half-maximal theory sits in an irreducible representation of $\mathrm{SO}(d, d)$, thus in a single irreducible representation of the $E_{d+1(d+1)}$ duality group of the maximal theory. It is thus less clear if the resulting gaugings sit in different orbits of the duality group according to their IIA/IIB origin, i.e. if IIA and IIB reductions give rise to inequivalent lower-dimensional theories. A natural starting point for further investigation are 3-dimensional maximal gauged SUGRAs. These are known to have two inequivalent $\mathrm{SO}(8)$ gaugings, expected to arise from $S^{7}$ reductions of IIA/IIB [53]. Indeed, the full EFT has been constructed for this case [54] so that the full reduction Ansätze of the $S^{7}$ truncations could then also be derived. It would also be interesting to cast into this framework consistent truncations of the massive IIA theory such as [55] which would require a (modest) dependence of the twist matrices on one of the non-physical coordinates, cf. [56].

Finally, it would be interesting to try and find a systematic procedure for the construction of the twist matrices for all possible allowed gaugings of the quadratic constraint (2.11). 
An interesting proposal for the case of half-maximal gauged SUGRA appeared in [20]. However, the resulting twist matrices are not $O(d, d)$-valued so that it is not immediately clear how to find the associated reduction Ansätze.

\section{Acknowledgments}

We would like to thank Michael Abbott, Charles Strickland-Constable, Olaf Hohm, Diego Marqués, Jose Fernández-Melgarejo and Alejandro Rosabal for helpful discussions. EM would like to thank the organisers of the Simons Summer Workshop 2015 and the CERNCKC TH Institute on Duality Symmetries in String and M-Theories for hospitality, while part of this work was being completed. EM is supported by the ERC Advanced Grant "Strings and Gravity" (Grant No. 32004) and was also funded by the National Research Foundation (NRF) of South Africa under grant CSUR13091742207 during the initial stages of this project.

Open Access. This article is distributed under the terms of the Creative Commons Attribution License (CC-BY 4.0), which permits any use, distribution and reproduction in any medium, provided the original author(s) and source are credited.

\section{References}

[1] M.J. Duff, B.E.W. Nilsson, C.N. Pope and N.P. Warner, On the Consistency of the Kaluza-Klein Ansatz, Phys. Lett. B 149 (1984) 90 [INSPIRE].

[2] B. de Wit and H. Nicolai, The Consistency of the $S^{7}$ Truncation in D $=11$ Supergravity, Nucl. Phys. B 281 (1987) 211 [inSPIRE].

[3] H. Nastase, D. Vaman and P. van Nieuwenhuizen, Consistency of the $A d S_{7} \times S^{4}$ reduction and the origin of selfduality in odd dimensions, Nucl. Phys. B 581 (2000) 179 [hep-th/9911238] [INSPIRE].

[4] M. Cvetič, H. Lü and C.N. Pope, Consistent Kaluza-Klein sphere reductions, Phys. Rev. D 62 (2000) 064028 [hep-th/0003286] [InSPIRE].

[5] G. Aldazabal, W. Baron, D. Marqués and C. Núñez, The effective action of Double Field Theory, JHEP 11 (2011) 052 [Erratum ibid. 11 (2011) 109] [arXiv:1109.0290] [INSPIRE].

[6] D. Geissbühler, Double Field Theory and $N=4$ Gauged Supergravity, JHEP 11 (2011) 116 [arXiv:1109.4280] [INSPIRE].

[7] D.S. Berman, E.T. Musaev and D.C. Thompson, Duality Invariant M-theory: Gauged supergravities and Scherk-Schwarz reductions, JHEP 10 (2012) 174 [arXiv:1208.0020] [INSPIRE].

[8] G. Aldazabal, M. Graña, D. Marqués and J.A. Rosabal, Extended geometry and gauged maximal supergravity, JHEP 06 (2013) 046 [arXiv: 1302.5419] [INSPIRE].

[9] K. Lee, C. Strickland-Constable and D. Waldram, Spheres, generalised parallelisability and consistent truncations, arXiv:1401.3360 [INSPIRE].

[10] F. Hassler and D. Lüst, Consistent Compactification of Double Field Theory on Non-geometric Flux Backgrounds, JHEP 05 (2014) 085 [arXiv:1401.5068] [INSPIRE]. 
[11] O. Hohm and H. Samtleben, Consistent Kaluza-Klein Truncations via Exceptional Field Theory, JHEP 01 (2015) 131 [arXiv:1410.8145] [INSPIRE].

[12] W. Cho, J.J. Fernández-Melgarejo, I. Jeon and J.-H. Park, Supersymmetric gauged double field theory: systematic derivation by virtue of twist, JHEP 08 (2015) 084 [arXiv: 1505.01301] [inSPIRE].

[13] A. Baguet, O. Hohm and H. Samtleben, Consistent Type IIB Reductions to Maximal 5D Supergravity, Phys. Rev. D 92 (2015) 065004 [arXiv:1506.01385] [INSPIRE].

[14] C.S. Shahbazi, A class of non-geometric M-theory compactification backgrounds, arXiv:1508.01750 [INSPIRE].

[15] N. Kaloper and R.C. Myers, The Odd story of massive supergravity, JHEP 05 (1999) 010 [hep-th/9901045] [INSPIRE].

[16] J. Shelton, W. Taylor and B. Wecht, Nongeometric flux compactifications, JHEP 10 (2005) 085 [hep-th/0508133] [INSPIRE].

[17] E.A. Bergshoeff, J. Gomis, T.A. Nutma and D. Roest, Kac-Moody Spectrum of (Half-)Maximal Supergravities, JHEP 02 (2008) 069 [arXiv:0711.2035] [INSPIRE].

[18] A. Le Diffon and H. Samtleben, Supergravities without an Action: Gauging the Trombone, Nucl. Phys. B 811 (2009) 1 [arXiv:0809.5180] [INSPIRE].

[19] G. Dibitetto, J.J. Fernández-Melgarejo, D. Marqués and D. Roest, Duality orbits of non-geometric fluxes, Fortsch. Phys. 60 (2012) 1123 [arXiv:1203.6562] [INSPIRE].

[20] P. du Bosque, F. Hassler and D. Lüst, Flux Formulation of DFT on Group Manifolds and Generalized Scherk-Schwarz Compactifications, arXiv:1509.04176 [INSPIRE].

[21] R. Blumenhagen, F. Hassler and D. Lüst, Double Field Theory on Group Manifolds, JHEP 02 (2015) 001 [arXiv: 1410.6374] [INSPIRE].

[22] H. Samtleben and M. Weidner, The Maximal $D=7$ supergravities, Nucl. Phys. B 725 (2005) 383 [hep-th/0506237] [INSPIRE].

[23] O. Hohm and H. Samtleben, Exceptional Form of D =11 Supergravity, Phys. Rev. Lett. 111 (2013) 231601 [arXiv:1308.1673] [INSPIRE].

[24] D.S. Berman and M.J. Perry, Generalized Geometry and M-theory, JHEP 06 (2011) 074 [arXiv: 1008.1763] [INSPIRE].

[25] C.D.A. Blair, E. Malek and J.-H. Park, M-theory and Type IIB from a Duality Manifest Action, JHEP 01 (2014) 172 [arXiv:1311.5109] [INSPIRE].

[26] O. Hohm and H. Samtleben, Exceptional Field Theory I: $E_{6(6)}$ covariant Form of M-theory and Type IIB, Phys. Rev. D 89 (2014) 066016 [arXiv:1312.0614] [InSPIRE].

[27] O. Hohm and H. Samtleben, Exceptional field theory. II. E $E_{7(7)}$, Phys. Rev. D 89 (2014) 066017 [arXiv: 1312.4542] [INSPIRE].

[28] H. Godazgar, M. Godazgar, O. Hohm, H. Nicolai and H. Samtleben, Supersymmetric $E_{7(7)}$ Exceptional Field Theory, JHEP 09 (2014) 044 [arXiv: 1406.3235] [INSPIRE].

[29] A. Coimbra, C. Strickland-Constable and D. Waldram, $E_{d(d)} \times \mathbb{R}^{+}$generalised geometry, connections and M-theory, JHEP 02 (2014) 054 [arXiv:1112.3989] [INSPIRE].

[30] A. Coimbra, C. Strickland-Constable and D. Waldram, Supergravity as Generalised Geometry II: $E_{d(d)} \times \mathbb{R}^{+}$and M-theory, JHEP 03 (2014) 019 [arXiv:1212.1586] [INSPIRE]. 
[31] E. Cremmer and B. Julia, The SO(8) Supergravity, Nucl. Phys. B 159 (1979) 141 [inSPIRE].

[32] D.S. Berman, H. Godazgar, M. Godazgar and M.J. Perry, The Local symmetries of M-theory and their formulation in generalised geometry, JHEP 01 (2012) 012 [arXiv:1110.3930] [INSPIRE].

[33] A. Abzalov, I. Bakhmatov and E.T. Musaev, Exceptional field theory: $\mathrm{SO}(5,5)$, JHEP 06 (2015) 088 [arXiv: 1504.01523] [INSPIRE].

[34] O. Hohm and Y.-N. Wang, Tensor hierarchy and generalized Cartan calculus in $\mathrm{SL}(3) \times \mathrm{SL}(2)$ exceptional field theory, JHEP 04 (2015) 050 [arXiv: 1501.01600] [INSPIRE].

[35] Y.-N. Wang, Generalized Cartan Calculus in general dimension, JHEP 07 (2015) 114 [arXiv: 1504 . 04780] [INSPIRE].

[36] J.-H. Park and Y. Suh, U-geometry: SL(5), JHEP 04 (2013) 147 [Erratum ibid. 11 (2013) 210] [arXiv: 1302.1652] [INSPIRE].

[37] C.D.A. Blair and E. Malek, Geometry and fluxes of SL(5) exceptional field theory, JHEP 03 (2015) 144 [arXiv:1412.0635] [INSPIRE].

[38] E.T. Musaev, Gauged supergravities in 5 and 6 dimensions from generalised Scherk-Schwarz reductions, JHEP 05 (2013) 161 [arXiv:1301.0467] [INSPIRE].

[39] D.S. Berman, C.D.A. Blair, E. Malek and M.J. Perry, The $O_{D, D}$ geometry of string theory, Int. J. Mod. Phys. A 29 (2014) 1450080 [arXiv:1303.6727] [INSPIRE].

[40] B. de Wit, H. Nicolai and H. Samtleben, Gauged Supergravities, Tensor Hierarchies and M-theory, JHEP 02 (2008) 044 [arXiv:0801.1294] [INSPIRE].

[41] W. Siegel, Superspace duality in low-energy superstrings, Phys. Rev. D 48 (1993) 2826 [hep-th/9305073] [INSPIRE].

[42] C. Hull and B. Zwiebach, Double Field Theory, JHEP 09 (2009) 099 [arXiv: 0904.4664] [INSPIRE].

[43] C. Hull and B. Zwiebach, The Gauge algebra of double field theory and Courant brackets, JHEP 09 (2009) 090 [arXiv:0908.1792] [INSPIRE].

[44] O. Hohm, C. Hull and B. Zwiebach, Background independent action for double field theory, JHEP 07 (2010) 016 [arXiv: 1003.5027] [INSPIRE].

[45] O. Hohm, C. Hull and B. Zwiebach, Generalized metric formulation of double field theory, JHEP 08 (2010) 008 [arXiv: 1006.4823] [INSPIRE].

[46] D.C. Thompson, Duality Invariance: From M-theory to Double Field Theory, JHEP 08 (2011) 125 [arXiv:1106.4036] [INSPIRE].

[47] K. Lee, C. Strickland-Constable and D. Waldram, New gaugings and non-geometry, arXiv: 1506.03457 [INSPIRE].

[48] G. Dibitetto, J.J. Fernández-Melgarejo and D. Marqués, All gaugings and stable de Sitter in $D=7$ half-maximal supergravity, JHEP 11 (2015) 037 [arXiv:1506.01294] [INSPIRE].

[49] O. Hohm and B. Zwiebach, Large Gauge Transformations in Double Field Theory, JHEP 02 (2013) 075 [arXiv: 1207.4198] [INSPIRE].

[50] J.-H. Park, Comments on double field theory and diffeomorphisms, JHEP 06 (2013) 098 [arXiv: 1304.5946] [INSPIRE]. 
[51] D.S. Berman, M. Cederwall and M.J. Perry, Global aspects of double geometry, JHEP 09 (2014) 066 [arXiv: 1401.1311] [INSPIRE].

[52] U. Naseer, A note on large gauge transformations in double field theory, JHEP 06 (2015) 002 [arXiv: 1504.05913] [INSPIRE].

[53] T. Fischbacher, H. Nicolai and H. Samtleben, Nonsemisimple and complex gaugings of $N=16$ supergravity, Commun. Math. Phys. 249 (2004) 475 [hep-th/0306276] [INSPIRE].

[54] O. Hohm and H. Samtleben, Exceptional field theory. III. E 8(8) $_{\text {, }}$ Phys. Rev. D 90 (2014) 066002 [arXiv:1406.3348] [INSPIRE].

[55] A. Guarino, D.L. Jafferis and O. Varela, String Theory Origin of Dyonic $N=8$ Supergravity and Its Chern-Simons Duals, Phys. Rev. Lett. 115 (2015) 091601 [arXiv:1504.08009] [INSPIRE].

[56] O. Hohm and S.K. Kwak, Massive Type II in Double Field Theory, JHEP 11 (2011) 086 [arXiv:1108.4937] [INSPIRE]. 